\title{
On the cyclotomic main conjecture for the prime 2
}

\author{
By Matthias Flach at Pasadena
}

\begin{abstract}
We complete the proof of the equivariant Tamagawa number conjecture for Tate motives over absolutely abelian fields by proving a refined cyclotomic main conjecture at the prime 2 .
\end{abstract}

\section{Introduction}

Let $l$ be a prime number, $m_{0}$ an integer prime to $l$ and

$$
\Lambda=\lim _{n} \mathbb{Z}_{l}\left[\operatorname{Gal}\left(\mathbb{Q}\left(\zeta_{m_{0} l^{n}}\right) / \mathbb{Q}\right)\right] \cong \lim _{n} \mathbb{Z}_{l}\left[\left(\mathbb{Z} / m_{0} l^{n} \mathbb{Z}\right)^{\times}\right]
$$

the cyclotomic Iwasawa algebra of "tame level $m_{0}$ ". Using étale cohomology one can define a certain perfect complex of $\Lambda$-modules $\Delta^{\infty}$ (see Section 1.2 below) and a certain basis $\mathscr{L}$ of the invertible $Q(\Lambda)$-module $\operatorname{Det}_{Q(\Lambda)}\left(\Delta^{\infty} \otimes_{\Lambda} Q(\Lambda)\right)$ where $Q(\Lambda)$ is the total ring of fractions of $\Lambda$. This basis $\mathscr{L}$ is obtained by $l$-adically interpolating the leading Taylor coefficients of the Dirichlet $L$-functions $L(\chi, s)$ at $s=0$ where $\chi$ runs through characters of conductors dividing $m_{0} l^{\infty}$. The main conjecture referred to in the title of this paper, Theorem 1.2 below, is the statement that $\mathscr{L}$ is in fact a $\Lambda$-basis of $\operatorname{Det}_{\Lambda} \Delta^{\infty}$.

This main conjecture was essentially proven for $l \neq 2$ by Burns and Greither [7] building on the theorem of Mazur and Wiles [15] (see also Rubin [18]) proving the traditional "Iwasawa main conjecture". The extra refinement in the theorem of Burns and Greither vis-à-vis the theorem of Mazur and Wiles lies in the fact that $\Lambda$ need not be a regular ring. Indeed if $l$ is odd then $\Lambda$ is regular if and only if $l \Varangle \phi\left(m_{0}\right)$ where $\phi$ is the Euler $\phi$-function.

In this article, we give a proof of Theorem 1.2 for $l=2$. This was claimed as Theorem 5.2 in the survey paper [9] but the proof given there, arguing separately for each height one prime $\mathfrak{q}$ of $\Lambda$, is incomplete at primes $\mathfrak{q}$ which contain $l=2$. The argument given in [9], p. 95, is an attempt to use only knowledge of the cohomology as well as perfectness of the complex $\Delta_{\mathfrak{q}}^{\infty}$ but it turns out that this information is insufficient. Here we shall use the techniques of the paper [6], such as the Coleman homomorphism and Leopoldt's result on

The author was supported by grant DMS-0701029 from the National Science Foundation. 
the Galois structure of cyclotomic integer rings, as well as Coleman's reciprocity law [8], Theorem 1, to construct the complex $\Delta_{\mathfrak{q}}^{\infty}$ more explicitly and thereby verify Theorem 1.2.

What is peculiar to the situation $l=2$ is not only that $\Lambda$ is never regular (due to the presence of the complex conjugation) but also that the $l$-adic $L$-function $\mathscr{L}$ is quite differently defined for even and odd characters. For even characters one interpolates first derivatives of Dirichlet $L$-functions via cyclotomic units, for odd characters one interpolates the values of these functions via Stickelberger elements. A proof of Theorem 1.2 therefore in some sense involves a "mod 2 congruence" between Stickelberger elements and cyclotomic units. Given the explicit nature of both objects, it is perhaps not surprising that this congruence turns out to be an elementary statement which is arrived at, however, only after some rather arduous computations. The statement is the following easy fact in the case $x=\frac{a}{m_{0} 2^{n}}, a \in \mathbb{Z}$ : Let $M \equiv 1 \bmod 4$ be an integer, $0<x<1$ a real number and $u=\exp (2 \pi i x)$. Then the sign of the real number $\frac{1-u^{M}}{(1-u)^{M}}$ is $(-1)^{\lfloor M x\rfloor}$. Here for any real
number $y$ we set $\lfloor y\rfloor=\max \{n \in \mathbb{Z} \mid n \leqq y\}$.

We believe that Theorem 1.2 (for any prime $l$ ) is the most precise statement one can make about the relation between leading Taylor coefficients of Dirichlet $L$-functions at $s=0$ and étale cohomology. It is a special case of very general conjectures on motivic $L$-values put forward by Kato [14] and Kato and Fukaya [10]. Theorem 1.2 for $l=2$ not only confirms Kato's point of view of $L$-values and $l$-adic $L$-functions as bases of determinant line bundles quite beautifully but also has other number theoretic consequences which were already noted in [9], 5.1, and [6], Corollaries 1.2 and 1.4. In particular, it completes the proof of the equivariant Tamagawa number conjecture for the motive $h^{0}(\operatorname{Spec}(L))(j)$ and the order $\mathbb{Z}[\operatorname{Gal}(L / \mathbb{Q})]$ where $j$ is any integer and $L / \mathbb{Q}$ any abelian extension. This includes the 2-primary part of the original (non-equivariant) Tamagawa number conjecture of Bloch and Kato [1] for the Riemann Zeta function. A consequence of the equivariant Tamagawa number conjecture is the validity of all three Chinburg conjectures asserting the vanishing of certain invariants $\Omega(L / K, i), i=1,2,3$, if $L / \mathbb{Q}$ is abelian (see [6], Corollary 1.4). For other consequences concerning Fitting ideals we refer to Greither's paper [12] and for the relevance to conjectures of Tate, Stark, Gross, Rubin, Popescu et al. to Burns' paper [3], Theorem A.

After recalling material from [9] and [6] in Section 1 we prove in Section 2 a statement which might be regarded as a functional equation of $\mathscr{L}$ and which is a refinement of a result going back to Iwasawa. We finally give the proof of Theorem 1.2 in Section 3 and collect some technical computations related to the Shapiro lemma in an appendix.

\section{Notation and preliminaries}

1.1. Cyclotomic fields. We follow the notation of [9] which we now recall. For any positive integer $m$ we set

$$
\begin{aligned}
\zeta_{m} & :=e^{2 \pi i / m}, \quad L_{m}:=\mathbb{Q}\left(\zeta_{m}\right), \\
\sigma_{m} & :=\text { the inclusion } L_{m} \rightarrow \mathbb{C}, \\
G_{m} & :=\operatorname{Gal}\left(L_{m} / \mathbb{Q}\right) \cong(\mathbb{Z} / m \mathbb{Z})^{\times} .
\end{aligned}
$$


For any Dirichlet character $\eta: G_{m} \rightarrow \mathbb{C}^{\times}$we denote by

$$
e_{\eta}=e_{\eta, m}=\frac{1}{\left|G_{m}\right|} \sum_{a \in(\mathbb{Z} / m \mathbb{Z})^{\times}} \eta(a)^{-1} \tau_{a, m}
$$

the corresponding idempotent, where $\tau_{a, m} \in G_{m}$ is defined by $\tau_{a, m}\left(\zeta_{m}\right)=\zeta_{m}^{a}$. Throughout this paper by a character we mean a (continuous) homomorphism from a (profinite) group to the unit group of a ring.

Lemma 1.1. For any $\mathbb{Q}$-rational (resp. $\mathbb{Q}_{l}$-rational) character $\chi$ of $G_{m}$ of conductor $f_{\chi} \mid m$, any $d \mid m$ and any primitive $d$-th root of unity $\zeta_{d}^{a}$ we have

$$
e_{\chi} \zeta_{d}^{a}= \begin{cases}0, & \text { if } f_{\chi} \nmid d \\ \frac{\phi\left(f_{\chi}\right)}{\phi(d)} \mu\left(\frac{d}{f_{\chi}}\right) \chi^{-1}\left(\frac{d}{f_{\chi}}\right) \chi(a) e_{\chi} \zeta_{f_{\chi}}, & \text { if } f_{\chi} \mid d\end{cases}
$$

in $L_{m}$ (resp. $\left.L_{m} \otimes_{\mathbb{Q}} \mathbb{Q}_{l}\right)$. Here $\phi(m)$ is Euler's $\phi$-function, $\mu(m)$ is the Möbius function, $\chi(a)=0$ if $\left(a, f_{\chi}\right)>1$ and we view a $\mathbb{Q}$-rational character $\chi$ as the tautological homomorphism

$$
G_{m} \rightarrow \mathbb{Q}\left[G_{m}\right]^{\times} \rightarrow\left(e_{\chi} \mathbb{Q}\left[G_{m}\right]\right)^{\times}=\mathbb{Q}(\chi)^{\times}
$$

where $e_{\chi}=\sum_{\eta \mapsto \chi} e_{\eta}$ (and similarly for $\mathbb{Q}_{l}$-rational characters $)$.

Proof. This is [6], Lemma 6.2.

For a prime number $l$ and integer $m_{0}$ prime to $l$, we define

$$
\begin{aligned}
& \ell= \begin{cases}l, & l \neq 2, \\
4, & l=2,\end{cases} \\
& G_{m_{0} l^{\infty}}:=\lim _{n} G_{m_{0} l^{n}}, \\
& \gamma:=1+\ell m_{0} \in G_{m_{0} l^{\infty}}, \\
& \Lambda:=\lim _{n} \mathbb{Z}_{l}\left[G_{m_{0} l^{n}}\right] \cong \mathbb{Z}_{l}\left[G_{m_{0} \ell}\right] \llbracket \gamma-1 \rrbracket .
\end{aligned}
$$

The Iwasawa algebra $\Lambda$ is a finite product of complete local 2-dimensional CohenMacaulay (even complete intersection) rings. However, it is regular if and only if $l \chi \# G_{\ell m_{0}}$. We denote by $Q(\Lambda)$ the total ring of fractions of $\Lambda$.

1.2. Global Iwasawa theory. Borrowing notation from [6] (and replacing $p$ by $l$ and setting $r=1)$ we define a free, rank one $\Lambda$-module with a continuous $\Lambda$-linear action of $\operatorname{Gal}(\overline{\mathbb{Q}} / \mathbb{Q})$,

$$
T_{l}^{\infty}=\lim _{n} H^{0}\left(\operatorname{Spec}\left(L_{m_{0} l^{n}} \otimes_{\mathbb{Q}} \overline{\mathbb{Q}}\right), \mathbb{Z}_{l}(1)\right)
$$


and a perfect complex of $\Lambda$-modules,

$$
\Delta^{\infty}=R \Gamma_{c}\left(\mathbb{Z}\left[\frac{1}{m_{0} l}\right], T_{l}^{\infty, *}(1)\right)^{*}[-3],
$$

where for any projective $\Lambda$-module $P$ (resp. perfect complex of $\Lambda$-modules $C$ ) we set $P^{*}=\operatorname{Hom}_{\Lambda}(P, \Lambda)\left(\right.$ resp. $\left.C^{*}=R \operatorname{Hom}_{\Lambda}(C, \Lambda)\right)$. The compact support étale cohomology is defined as in [5], p. 522. We recall the computation of the cohomology of $\Delta^{\infty}$ from [9]. We have $H^{i}\left(\Delta^{\infty}\right)=0$ for $i \neq 1,2$, a canonical, isomorphism

$$
H^{1}\left(\Delta^{\infty}\right) \cong U_{\left\{v \mid m_{0} l\right\}}^{\infty}:=\lim _{n} \mathcal{O}_{L_{m_{0} l n}}\left[\frac{1}{m_{0} l}\right]^{\times} \otimes_{\mathbb{Z}} \mathbb{Z} l
$$

and a short exact sequence

$$
0 \rightarrow P_{\left\{v \mid m_{0} l\right\}}^{\infty} \rightarrow H^{2}\left(\Delta^{\infty}\right) \rightarrow X_{\left\{v \mid m_{0} l \infty\right\}}^{\infty} \rightarrow 0
$$

where

$$
\begin{aligned}
& P_{\left\{v \mid m_{0} l\right\}}^{\infty}:=\lim _{n} \operatorname{Pic}\left(\mathcal{O}_{L_{m_{0} l n}}\left[\frac{1}{m_{0} l}\right]\right) \otimes_{\mathbb{Z}} \mathbb{Z}_{l}, \\
& X_{\left\{v \mid m_{0} l \infty\right\}}^{\infty}:=\lim _{n} X_{\left\{v \mid m_{0} l \infty\right\}}\left(L_{m_{0} l^{n}}\right) \otimes_{\mathbb{Z}} \mathbb{Z}_{l} .
\end{aligned}
$$

For any number field $L$ and set of places $S$ of $L$ we set here

$$
Y_{S}(L):=\bigoplus_{v \in S} \mathbb{Z}, \quad X_{S}(L):=\left\{\left(a_{v}\right) \in Y_{S}(L) \mid \sum_{v} a_{v}=0\right\},
$$

and all limits are taken with respect to Norm maps (on $Y_{S}$ this is the map sending a place to its restriction). For $d \mid m_{0}$ put

$$
\begin{aligned}
\eta_{d} & :=\left(1-\zeta_{\ell d l^{n}}\right)_{n \geqq 0} \in U_{\left\{v \mid m_{0} l\right\}}^{\infty}, \\
\sigma & :=\left(\sigma_{\ell m_{0} l^{n}}\right)_{n \geqq 0} \in Y_{\left\{v \mid m_{0} l \infty\right\}}^{\infty}, \\
\theta_{d} & :=\left(g_{\ell d l^{n}}\right)_{n \geqq 0} \in \frac{1}{\left[L_{m_{0}}: L_{d}\right]} \cdot\left(\gamma-\chi_{\text {cyclo }}(\gamma)\right)^{-1} \Lambda,
\end{aligned}
$$

where

$$
g_{k}=-\sum_{\substack{0<a<k \\(a, k)=1}}\left(\frac{a}{k}-\frac{1}{2}\right) \tau_{a, k}^{-1} \in \mathbb{Q}\left[G_{k}\right]
$$

with $\tau_{a, k} \in G_{k}$ defined by $\tau_{a, k}\left(\zeta_{k}\right)=\zeta_{k}^{a}$. Here we also view $\tau_{a, k}$ as an element of $\mathbb{Q}\left[G_{k^{\prime}}\right]$ for $k \mid k^{\prime}$ (which allows us to view $\theta_{d}$ as an element of the fraction field of $\Lambda$ for $d \mid m_{0}$ ) by 
$\tau_{a, k} \mapsto\left[G_{k^{\prime}}: G_{k}\right]^{-1} \sum_{a^{\prime} \equiv a \bmod k} \tau_{a^{\prime}, k^{\prime}}$. The relationship between $\theta_{d}$ and $l$-adic $L$-functions in the usual normalization is given by the interpolation formula

$$
\chi \chi_{\text {cyclo }}^{j}\left(\theta_{d}\right)=\left(1-\chi^{-1}(l) l^{-j}\right) L\left(\chi^{-1}, j\right)=: L_{l}\left(\chi^{-1} \omega^{1-j}, j\right)
$$

for all Dirichlet characters $\chi$ of conductor $d l^{n}$ and $j \leqq 0$ (here $\omega$ and $\chi_{\text {cyclo denote the }}$ Teichmueller and cyclotomic character $G_{m_{0}} l^{\infty} \rightarrow \mathbb{Z}_{l}^{\times}$, respectively).

We fix an embedding $\overline{\mathbb{Q}}_{l} \rightarrow \mathbb{C}$ and identify $\hat{G}_{k}$ with the set of $\overline{\mathbb{Q}}_{l}$-valued characters. The total ring of fractions

$$
Q(\Lambda) \cong \prod_{\psi \in \hat{G}_{\ell m_{0}}^{\mathbb{Q}_{l}}} Q(\psi)
$$

of $\Lambda$ is a product of fields indexed by the $\mathbb{Q}_{l}$-rational characters $\psi$ of $G_{\ell m_{0}}$. As in [9] one easily computes

$$
\operatorname{dim}_{Q(\psi)}\left(U_{\left\{v \mid m_{0} l\right\}}^{\infty} \otimes_{\Lambda} Q(\psi)\right)=\operatorname{dim}_{Q(\psi)}\left(Y_{\left\{v \mid m_{0} l \infty\right\}}^{\infty} \otimes_{\Lambda} Q(\psi)\right)= \begin{cases}1, & \psi \text { even }, \\ 0, & \psi \text { odd } .\end{cases}
$$

Note that the inclusion $X_{\left\{v \mid m_{0} l \infty\right\}}^{\infty} \subseteq Y_{\left\{v \mid m_{0} l \infty\right\}}^{\infty}$ becomes an isomorphism after tensoring with $Q(\psi)$ and that $e_{\psi}\left(\eta_{m_{0}}^{-1} \otimes \sigma\right)$ is a $Q(\psi)$-basis of

$$
\begin{aligned}
\operatorname{Det}_{Q(\psi)}^{-1}\left(U_{\left\{v \mid m_{0} l\right\}}^{\infty}\right. & \left.\otimes_{\Lambda} Q(\psi)\right) \otimes \operatorname{Det}_{Q(\psi)}\left(X_{\left\{v \mid m_{0} l \infty\right\}}^{\infty} \otimes_{\Lambda} Q(\psi)\right) \\
& \cong \operatorname{Det}_{Q(\psi)}\left(\Delta^{\infty} \otimes_{\Lambda} Q(\psi)\right)
\end{aligned}
$$

for even $\psi$. For odd $\psi$ the complex $\Delta^{\infty} \otimes_{\Lambda} Q(\psi)$ is acyclic and we can view $e_{\psi} \theta_{m_{0}} \in Q(\psi)$ as an element of

$$
\operatorname{Det}_{Q(\psi)}\left(\Delta^{\infty} \otimes_{\Lambda} Q(\psi)\right) \cong Q(\psi) .
$$

Note also that $e_{\psi} \theta_{m_{0}}=0$ (resp. $\left.e_{\psi}\left(\eta_{m_{0}}^{-1} \otimes \sigma\right)=0\right)$ if $\psi$ is even (resp. odd). Hence, we obtain a $Q(\Lambda)$-basis

$$
\mathscr{L}:=\theta_{m_{0}}^{-1}+2 \cdot \eta_{m_{0}}^{-1} \otimes \sigma \in \operatorname{Det}_{Q(\Lambda)}\left(\Delta^{\infty} \otimes_{\Lambda} Q(\Lambda)\right)
$$

of the invertible $Q(\Lambda)$-module $\operatorname{Det}_{Q(\Lambda)}\left(\Delta^{\infty} \otimes_{\Lambda} Q(\Lambda)\right)$.

Theorem 1.2. There is an equality of invertible $\Lambda$-submodules

$$
\Lambda \cdot \mathscr{L}=\operatorname{Det}_{\Lambda} \Delta^{\infty}
$$

of $\operatorname{Det}_{Q(\Lambda)}\left(\Delta^{\infty} \otimes_{\Lambda} Q(\Lambda)\right)$.

For odd primes $l$ this theorem is essentially due to Burns and Greither [7] and a proof of this precise statement with this precise notation was given in [9], Section 5.1. For $l=2$ the theorem is new and its proof will occupy Section 3 of the present paper. 
1.3. Local Iwasawa theory. Here we recall results from [6]. The cohomology of $R \Gamma\left(\mathbb{Q}_{l}, T_{l}^{\infty}\right)$ is naturally isomorphic to

$$
H^{i}\left(\mathbb{Q}_{l}, T_{l}^{\infty}\right) \cong \begin{cases}\lim _{n} \mathbb{Q}\left(\zeta_{m_{0} l^{n}}\right)_{l}^{\times} / l^{n}, & i=1, \\ \prod_{v \mid l} \mathbb{Z}_{l}, & i=2, \\ 0, & \text { otherwise }\end{cases}
$$

where $\mathbb{Q}\left(\zeta_{m_{0} l^{n}}\right)_{l}:=\mathbb{Q}\left(\zeta_{m_{0} l^{n}}\right) \otimes_{\mathbb{Q}} \mathbb{Q}_{l}$ is a finite product of local fields and the limit is taken with respect to the norm maps. The valuation map induces a natural short exact sequence

$$
0 \longrightarrow U_{\mathrm{loc}}^{\infty}:=\lim _{n} \mathcal{O}_{\stackrel{\mathbb{Q}}{(}\left(\zeta_{m_{0} l^{n}}\right)_{l}} / l^{n} \longrightarrow \underset{n}{\lim } \mathbb{Q}\left(\zeta_{m_{0} l^{n}}\right)_{l}^{\times} / l^{n} \stackrel{\mathrm{val}}{\longrightarrow} \prod_{v \mid l} \mathbb{Z}_{l} \longrightarrow 0
$$

and in addition Coleman has constructed an exact sequence [17], Proposition 4.1.3,

$$
0 \rightarrow \prod_{v \mid l} \mathbb{Z}_{l}(1) \rightarrow U_{\mathrm{loc}}^{\infty} \stackrel{\Theta}{\rightarrow} R \rightarrow \prod_{v \mid l} \mathbb{Z}_{l}(1) \rightarrow 0
$$

where

$$
R:=\left\{f \in \mathbb{Z}\left[\zeta_{m_{0}}\right]_{l} \llbracket X \rrbracket \mid \psi(f):=\sum_{\zeta^{l}=1} f(\zeta(1+X)-1)=0\right\}
$$

and $\mathbb{Z}\left[\zeta_{m_{0}}\right]_{l}$ denotes the finite étale $\mathbb{Z}_{l}$-algebra $\mathbb{Z}\left[\zeta_{m_{0}}\right] \otimes_{\mathbb{Z}} \mathbb{Z}_{l}$. Moreover, the map $\Theta$ is given by

$$
\Theta(u)=\left(1-\frac{\phi}{l}\right) \log \left(f_{u}\right),
$$

where $f_{u}$ is the (unique) Coleman power series of the norm compatible system of units $u$ with respect to $\left(\zeta_{l^{n}}\right)_{n \geqq 1}$ and one has $\phi(f)(X)=f^{\mathrm{Fr}_{l}}\left((1+X)^{l}-1\right)$. The $\mathbb{Z}_{l}$-module $R$ carries a natural continuous $G_{m_{0}} l^{\infty}$-action [17], 1.1.4, and with respect to this action all maps in (6), (7) and (8) are $\Lambda$-equivariant.

Lemma 1.3. The $\Lambda$-module $R$ is free of rank one with basis

$$
\beta_{m_{0}}^{\infty}:=\xi_{m_{0}}(1+X), \quad \xi_{m_{0}}:=\sum_{m_{1}|d| m_{0}} \zeta_{d}
$$

where $m_{1}=\prod_{p \mid m_{0}} p$.

Proof. This is [6], Lemma 5.1.

Proposition 1.4. Viewing $\beta_{m_{0}}^{\infty}$ as a $Q(\Lambda)$-basis of

$$
\begin{aligned}
R \otimes_{\Lambda} Q(\Lambda) & \cong U_{\mathrm{loc}}^{\infty} \otimes_{\Lambda} Q(\Lambda) \cong H^{1}\left(\mathbb{Q}_{l}, T_{l}^{\infty}\right) \otimes_{\Lambda} Q(\Lambda) \\
& \cong\left(\operatorname{Det}_{\Lambda}^{-1} R \Gamma\left(\mathbb{Q}_{l}, T_{l}^{\infty}\right)\right) \otimes_{\Lambda} Q(\Lambda)
\end{aligned}
$$


one has

$$
\Lambda \cdot \beta_{m_{0}}^{\infty}=\operatorname{Det}_{\Lambda}^{-1} R \Gamma\left(\mathbb{Q}_{l}, T_{l}^{\infty}\right) \subset\left(\operatorname{Det}_{\Lambda}^{-1} R \Gamma\left(\mathbb{Q}_{l}, T_{l}^{\infty}\right)\right) \otimes_{\Lambda} Q(\Lambda)
$$

Proof. This is [6], Proposition 5.2.

The following proposition is an application of the reciprocity law of Coleman [8], Theorem 1, and is not contained in [6]. We note that Coleman's reciprocity law includes the case $l=2$. Perrin-Riou's proof of her reciprocity law [17], Conjecture 3.6.4, in the special case of an $l$-adic representation $V=V_{0}(1), V_{0}$ unramified, in [17], Theorem 4.3.2, is a similar application of Coleman's result.

The cup-product pairing

$$
R \Gamma\left(\mathbb{Q}_{l}, T_{l}^{\infty}\right) \times R \Gamma\left(\mathbb{Q}_{l}, T_{l}^{\infty, *}(1)\right) \rightarrow R \Gamma\left(\mathbb{Q}_{l}, \Lambda(1)\right) \rightarrow \Lambda[-2]
$$

induces a pairing

$$
R \Gamma\left(\mathbb{Q}_{l}, T_{l}^{\infty}\right) \otimes_{\Lambda}^{L} R \Gamma\left(\mathbb{Q}_{l}, T_{l}^{\infty, *}(1)\right) \rightarrow \Lambda[-2]
$$

and a pairing on determinants

$$
\operatorname{Det}_{\Lambda}^{-1} R \Gamma\left(\mathbb{Q}_{l}, T_{l}^{\infty}\right) \otimes_{\Lambda} \operatorname{Det}_{\Lambda}^{-1} R \Gamma\left(\mathbb{Q}_{l}, T_{l}^{\infty, *}(1)\right) \rightarrow \Lambda
$$

which is perfect by local Tate duality, i.e. the arrow is an isomorphism. Denote by $\kappa^{\#}: G_{m_{0} l^{\infty}} \rightarrow \Lambda^{\times}$the character

$$
\kappa^{\#}: g \mapsto \chi_{\text {cyclo }}(g) g^{-1}
$$

as well as the induced ring automorphism of $\Lambda$. Note that there is a natural isomorphism of $\operatorname{Gal}(\overline{\mathbb{Q}} / \mathbb{Q})$-modules

$$
T_{l}^{\infty, *}(1) \cong T_{l}^{\infty} \otimes_{\Lambda, \kappa^{\#}} \Lambda
$$

which induces an isomorphism of perfect complexes of $\Lambda$-modules

$$
R \Gamma\left(\mathbb{Q}_{l}, T_{l}^{\infty, *}(1)\right) \cong R \Gamma\left(\mathbb{Q}_{l}, T_{l}^{\infty}\right) \otimes_{\Lambda, \kappa^{\#}} \Lambda
$$

Hence we may regard the pairing $(10)$ as a perfect $\kappa^{\#}$-sesquilinear pairing $\langle-,-\rangle$ on $\operatorname{Det}_{\Lambda}^{-1} R \Gamma\left(\mathbb{Q}_{l}, T_{l}^{\infty}\right)$. Recall that $\beta_{m_{0}}^{\infty}$ is a basis of this last module by Proposition 1.4. Let

$$
\varrho:=\left\langle\beta_{m_{0}}^{\infty}, \beta_{m_{0}}^{\infty}\right\rangle \in \Lambda^{\times}
$$

be the discriminant of this pairing, let $c \in G_{m_{0}} l^{\infty}$ be the complex conjugation and $c_{l}$ the projection of $c$ into the direct factor $G_{\ell}$ of $G_{m_{0}} l^{\infty}$. 
Proposition 1.5. The element $\varrho$ lies in $\mathbb{Z}_{l}\left[G_{m_{0} l^{\infty}}^{\text {tor }}\right]=\mathbb{Z}_{l}\left[G_{m_{0} \ell}\right]$ and we have $\varrho=\varrho_{0} c_{l}$ where $\varrho_{0}=\sum_{g \in G_{m_{0}}} \varrho_{0}(g) g^{-1} \in \mathbb{Z}_{l}\left[G_{m_{0}}\right]$ is given by

$$
\varrho_{0}(g)=\sum_{m_{1}|d|\left((g+1) m_{1}, m_{0}\right)} \frac{\phi\left(m_{0}\right)}{\phi(d /(d, g+1))} \mu\left(\frac{d}{(d, g+1)}\right) .
$$

Here we view $g \in G_{m_{0}}$ as an integer prime to $m_{0}$, and the summation is over all multiples $d$ of $m_{1}$ which also divide the g.c.d. $\left((g+1) m_{1}, m_{0}\right)$ of $(g+1) m_{1}$ and $m_{0}$.

Proof. The cup product also induces the $\kappa^{\#}$-sesquilinear pairing in the top row of the following diagram:

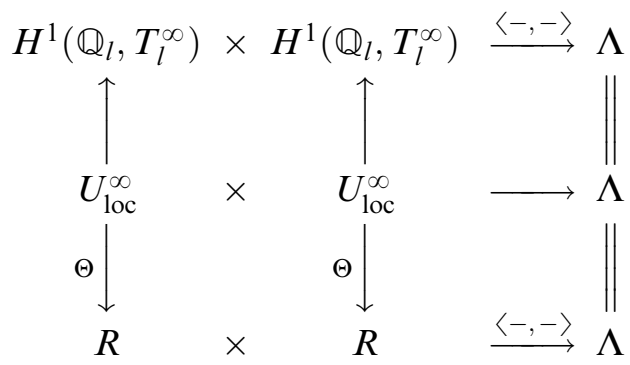

and Coleman's reciprocity law will allow us to give an explicit pairing in the bottom row that makes the diagram commutative. Note that

$$
H^{1}\left(\mathbb{Q}_{l}, T_{l}^{\infty}\right) \cong \lim _{n} H^{1}\left(\mathbb{Q}\left(\zeta_{m_{0} l^{n}}\right)_{l}, \mu_{l^{n}}\right)
$$

and for $u, v \in H^{1}\left(\mathbb{Q}_{l}, T_{l}^{\infty}\right)$, we have

$$
\langle u, v\rangle \equiv \sum_{g \in G_{m_{0} l^{n}}}\left\langle u_{n}^{g}, v_{n}\right\rangle_{n} g^{-1} \text { in } \mathbb{Z} / l^{n} \mathbb{Z}\left[G_{m_{0} l^{n}}\right]
$$

where the Hilbert symbol

$$
\langle-,-\rangle_{n}: H^{1}\left(\mathbb{Q}\left(\zeta_{m_{0} l^{n}}\right)_{l}, \mu_{l^{n}}\right) \times H^{1}\left(\mathbb{Q}\left(\zeta_{m_{0} l^{n}}\right)_{l}, \mu_{l^{n}}\right) \rightarrow \mathbb{Z} / l^{n} \mathbb{Z}
$$

is defined by $x \cup y=\zeta_{l^{n}}^{\langle x, y\rangle_{n}}$. For $u, v \in U_{\text {loc }}^{\infty}$ Coleman's reciprocity law [8], Theorem 1, computes the Hilbert symbol as

$$
\left\langle u_{n}, v_{n}\right\rangle_{n} \equiv \operatorname{Tr}_{\mathbb{Q}\left(\zeta_{m_{0}}\right)_{l} / \mathbb{Q}_{l}}\left(l^{-n} \sum_{\zeta \in \mu_{l} n} \Theta(u)(\zeta-1) D\left(\log f_{v}\right)(\zeta-1)\right) \bmod l^{n}
$$

where $D$ is the differential operator $D=(1+X) \frac{d}{d X}$. Since

$$
\sum_{\zeta \in \mu_{l} n} F(\zeta-1) D\left(\log f_{v}\right)(\zeta-1)=\sum_{\zeta \in \mu_{l}} F(\zeta-1) D(\Theta(v))(\zeta-1)
$$


by the argument in [17], at the bottom of page 146, we conclude that the pairing $\langle-,-\rangle$ on $R$ given by

$$
\langle F, G\rangle \equiv \sum_{g \in G_{m_{0} l^{n}}}\left\langle F^{g}, G\right\rangle_{n} g^{-1} \text { in } \mathbb{Z} / l^{n} \mathbb{Z}\left[G_{m_{0} l^{n}}\right],
$$

where

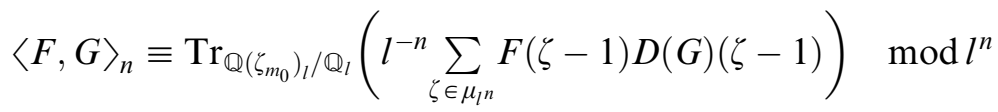

makes the above diagram commutative. Note here that the action of $g \in G_{m_{0} l^{n}}$ on $F \in R$ is independent of the lift of $g$ to $G_{m_{0} l^{\infty}}$ modulo $\gamma^{l^{n-1}}-1\left(\gamma^{2^{n-2}}-1\right.$ if $\left.l=2\right)$. Taking $F=G=\beta_{m_{0}}^{\infty}=\xi_{m_{0}}(1+T)$, we have $D(G)=G$ and

$$
\begin{aligned}
\left\langle\beta_{m_{0}}^{\infty, g}, \beta_{m_{0}}^{\infty}\right\rangle_{n} & \equiv \operatorname{Tr}_{\left.\mathbb{Q}_{\left(\zeta_{0}\right.}\right)_{l} / \mathbb{Q}_{l}}\left(l^{-n} \sum_{\zeta \in \mu_{l} n} \xi_{m_{0}}^{g} \zeta^{g} \xi_{m_{0}} \zeta\right) \bmod l^{n} \\
& \equiv \begin{cases}\operatorname{Tr}_{\mathbb{Q}_{(}\left(\zeta_{m_{0}}\right)_{l} / \mathbb{Q}_{l}}\left(\xi_{m_{0}}^{1+g}\right), & \text { if } g \equiv-1 \bmod l^{n} \\
0, & \text { otherwise. }\end{cases}
\end{aligned}
$$

Now by Lemma 1.1

$$
\begin{aligned}
\operatorname{Tr}_{\mathbb{Q}\left(\zeta_{m_{0}}\right)_{l} / \mathbb{Q}_{l}}\left(\xi_{m_{0}}^{1+g}\right) & =\phi\left(m_{0}\right) e_{1} \xi_{m_{0}}^{1+g} \\
& =\phi\left(m_{0}\right) \sum_{d, d^{\prime}} e_{1} \zeta_{D / f}^{D / d^{\prime} f+g D / d f}=\sum_{d, d^{\prime}} \frac{\phi\left(m_{0}\right)}{\phi(D / f)} \mu(D / f) \cdot 1
\end{aligned}
$$

where $d, d^{\prime}$ run through all multiples of $m_{1}$ dividing $m_{0}, \quad D=1 . c . m .\left(d, d^{\prime}\right)$ and $f=$ g.c.d. $\left(D / d^{\prime}+g D / d, D\right)$. Here we view $g$ as an integer prime to $m_{0}$. Now if $d \neq d^{\prime}$ then there exists a prime $p$ dividing $D / d^{\prime}$ or $D / d$ but not both since $D$ is the least common multiple. Hence $p \nmid f$. Also, $p^{2} \mid D$ since $p \mid d$ and $p \mid d^{\prime}$ in any case. Hence $p^{2} \mid D / f$ and $\mu(D / f)=0$. So the sum (14) reduces to the diagonal

$$
\operatorname{Tr}_{\mathbb{Q}_{(}\left(\zeta_{m_{0}}\right)_{l} / \mathbb{Q}_{l}}\left(\xi_{m_{0}}^{1+g}\right)=\sum_{m_{1}|d| m_{0}} \frac{\phi\left(m_{0}\right)}{\phi(d / f)} \mu(d / f)=\sum_{m_{1}|d|\left((g+1) m_{1}, m_{0}\right)} \frac{\phi\left(m_{0}\right)}{\phi(d / f)} \mu(d / f),
$$

which in turn reduces to a sum over $d$ so that $d / f=d /(d, g+1)$ is squarefree. This happens if and only if $d \mid(g+1) m_{1}$. In summary then

$$
\varrho=\left\langle\beta_{m_{0}}^{\infty}, \beta_{m_{0}}^{\infty}\right\rangle=\left(\sum_{g \in G_{m_{0} l^{n}}}\left\langle\beta_{m_{0}}^{\infty, g}, \beta_{m_{0}}^{\infty}\right\rangle_{n} g^{-1}\right)_{n}=\varrho_{0} c_{l}
$$

where $\varrho_{0}$ was defined in $(13)$.

\section{The functional equation}

In this section, we prove a result, Proposition 2.2 below, which is a key ingredient in the proof of Theorem 1.2 for $l=2$ but which is valid for all primes $l$ and which is a more precise form of a theorem going back to Iwasawa ("the $l$-adic $L$-function is the character- 
istic ideal of local units modulo cyclotomic units"). Proposition 2.2 can also be viewed as some kind of functional equation of the $l$-adic $L$-function $\mathscr{L}$ as we shall explain at the end of this section.

For any subgroup $H \subseteq G_{m_{0}} \subset G_{m_{0} l^{\infty}}^{\text {tor }}$ we set

$$
e_{H}=\sum_{\chi(H)=1} e_{\chi}=\frac{1}{|H|} \sum_{g \in H} g,
$$

where the sum is over all $\mathbb{Q}_{l}$-rational characters that vanish on $H$. For any prime $p \neq l$ we denote by

$$
I_{p} \subseteq G_{m_{0}} \subset G_{m_{0} l^{\infty}}^{\mathrm{tor}} \subseteq \Lambda^{\times}
$$

the inertia subgroup at $p$. For $x \in \Lambda$ we set

$$
e_{p}(x)=1+(x-1) e_{I_{p}} \in \Lambda\left[\frac{1}{l}\right]
$$

so that $x \mapsto e_{p}(x)$ is a multiplicative map. We denote by $\operatorname{Fr}_{p} \in G_{m_{0} l^{\infty}}\left(\operatorname{resp} . \widetilde{\operatorname{Fr}}_{p} \in G_{m_{0} l^{\infty}}\right)$ the choice of a Frobenius element with trivial component in $I_{p}\left(\operatorname{resp} . G_{m_{0}}\right)$. Note here that $I_{p}$ and $G_{m_{0}}$ are canonically direct factors of $G_{m_{0} l^{\infty}}$ by the Chinese remainder theorem.

Lemma 2.1. Fix a prime $p$ that is not equal to $l$. If $u: \mathbb{Z} \rightarrow \Lambda^{\times}$is any function such that $p-1$ divides $u(0)-u(1)$ in $\Lambda$, then $\sum_{\chi} u\left(\operatorname{ord}_{p}\left(f_{\chi}\right)\right) e_{\chi, m_{0}}$ is a unit of $\Lambda$ where the sum is
over all $\mathbb{Q}_{l}$-rational characters of $G_{m_{0}}$.

Proof. This is [6], Lemma 4.5, with $\mathbb{Z}_{l}[G]$ replaced by $\Lambda$. The proof of this lemma in loc. cit. transfers verbatim (with the roles of $l$ and $p$ interchanged).

Proposition 2.2. Using Lemma 2.1 define $v_{p} \in \Lambda^{\times}$via the function

$$
x \mapsto\left(p / \widetilde{\operatorname{Fr}}_{p}\right)^{\max (x, 1)}
$$

With the notation of (2), (9), (11) and Lemma 1.3 one has

$$
\Theta\left(\eta_{m_{0}}\right)=-\frac{1}{m_{0}} \prod_{p \mid m_{0}}\left(\frac{e_{p}\left(1-\mathrm{Fr}_{p}\right)}{e_{p}\left(1-\frac{\mathrm{Fr}_{p}}{p}\right)} v_{p}\right) \kappa^{\#}\left(\theta_{m_{0}}\right) \cdot \beta_{m_{0}}^{\infty},
$$

if $m_{0}>1$ and

$$
\Theta\left((\gamma-1) \eta_{m_{0}}\right)=-(\gamma-1) \kappa^{\#}\left(\theta_{m_{0}}\right) \cdot \beta_{m_{0}}^{\infty}
$$

if $m_{0}=1$.

Remarks. (a) If $\Lambda$ is regular, equivalently if $l \Varangle \phi\left(\ell m_{0}\right)$, this identity, up to an unspecified unit in $\Lambda$, is essentially due to Gillard [11], Theorem 1, and goes back to Iwasawa [13], see also [19], Theorem 13.56, for $m_{0}=1$ and $l \neq 2$. However, we need an exact formula in our later applications. 
(b) Following the point of view that $\mathscr{L}$ is an $l$-adic $L$-function with Euler factors at primes dividing $m_{0}$ removed, one might be tempted to regard the factor

$$
\prod_{p \mid m_{0}} \frac{e_{p}\left(1-\mathrm{Fr}_{p}\right)}{e_{p}\left(1-\frac{\mathrm{Fr}_{p}}{p}\right)}
$$

as the quotient of the missing Euler factors of $\eta_{m_{0}}$ and $\theta_{m_{0}}$. This is, however, not quite correct since the Euler factor of $\eta$ is not $1-\operatorname{Fr}_{p}$ but $1-\operatorname{Fr}_{p}^{-1}$, i.e. the projection of $\eta_{m_{0}}$ into $U_{\left\{v \mid m_{0} l\right\}}^{\infty} \otimes_{\Lambda} \Lambda^{\prime}$ is $\left(1-\mathrm{Fr}_{p}^{-1}\right) \eta_{m_{0}^{\prime}}$ where $m_{0}=m_{0}^{\prime} p^{\operatorname{ord}_{p}\left(m_{0}\right)}$. One cannot absorb this difference into the unit $v_{p}$ by introducing the factor $-\mathrm{Fr}_{p}=\left(1-\mathrm{Fr}_{p}\right) /\left(1-\mathrm{Fr}_{p}^{-1}\right)$ since $e_{p}\left(-\mathrm{Fr}_{p}\right) \notin \Lambda^{\times}$. Nonetheless, the formula in Proposition 2.2 is correct. One can verify that it is compatible with projection from level $m_{0}$ to level $m_{0}^{\prime}$, and it is also compatible with Lemma 2.5 below.

Proof. We first assume $m_{0}>1$. Let

$$
f(X):=1-\zeta_{m_{0}}(1+X)^{m_{0}^{-1}}
$$

be the Coleman power series for $\eta_{m_{0}}$ and define $g(X) \in \mathbb{Z}\left[\zeta_{m_{0}}\right]_{l} \llbracket X \rrbracket$ by

$$
g(X):=\Theta\left(\eta_{m_{0}}\right)=\left(1-l^{-1} \phi\right) \log (f(X))=\lambda \cdot \beta_{m_{0}}^{\infty}
$$

where this last equality defines $\lambda \in \Lambda$ via Lemma 1.3. By a standard result for the Amice transform [17], Lemma 1.1.6(ii), one has for $k \geqq 1$

$$
\chi_{\mathrm{cyclo}}^{k}(\lambda) \cdot \xi_{m_{0}}=\left(D^{k} g\right)(0) \in \mathbb{Z}\left[\zeta_{m_{0}}\right]_{l}
$$

where $D$ denotes again the differential operator $D=(1+X) \frac{d}{d X}$. Since $D \phi=l \phi D$ one has

$$
\begin{aligned}
D^{k} g(X) & =D^{k}\left(1-l^{-1} \phi\right) \log (f(X))=\left(1-\phi l^{k-1}\right) D^{k} \log (f(X)) \\
& =\left(1-\phi l^{k-1}\right) D^{k-1}(1+X) \frac{f^{\prime}(X)}{f(X)} \\
& =\left(1-\phi l^{k-1}\right) D^{k-1} \frac{-\zeta_{m_{0}} m_{0}^{-1}(1+X)^{m_{0}^{-1}}}{1-\zeta_{m_{0}}(1+X)^{m_{0}^{-1}}} \\
& =\left(1-\phi l^{k-1}\right)\left(\frac{d}{d z}\right)^{k-1} \frac{\zeta_{m_{0}} m_{0}^{-1} e^{z / m_{0}}}{\zeta_{m_{0}} e^{z / m_{0}}-1} \\
& =\left(1-\phi l^{k-1}\right)\left(\frac{d}{d z}\right)^{k-1}\left(\frac{1}{m_{0}} \sum_{a=1}^{m_{0}} \zeta_{m_{0}}^{a} \frac{e^{a z / m_{0}}}{e^{z}-1}\right) \\
& =\left(1-\phi l^{k-1}\right) \frac{1}{m_{0}}\left(\frac{d}{d z}\right)^{k-1} \sum_{a=1}^{m_{0}} \zeta_{m_{0}}^{a}\left(\frac{1}{z}+\sum_{n=1}^{\infty} \frac{B_{n}\left(\frac{a}{m_{0}}\right)}{n} \frac{z^{n-1}}{(n-1) !}\right)
\end{aligned}
$$


where $X=e^{z}-1$, so $D=\frac{d}{d z}$ and $B_{n}(t) \in \mathbb{Q}[t]$ is the Bernoulli polynomial. Note that $\sum_{a=1}^{m_{0}} \zeta_{m_{0}}^{a} \frac{1}{z}=0$. Taking $X=0$, or equivalently $z=0$, we find

$$
\left(D^{k} g\right)(0)=\left(1-\mathrm{Fr}_{l} l^{k-1}\right) \frac{1}{m_{0}} \sum_{a=1}^{m_{0}} \zeta_{m_{0}}^{a} \frac{B_{k}\left(\frac{a}{m_{0}}\right)}{k} .
$$

Let $\chi$ be a $\mathbb{Q}_{l}$-rational character of $G_{m_{0}}$ and $e_{\chi}=e_{\chi, m_{0}}$ the corresponding idempotent. Then Lemma 1.1 together with Lemma 2.3 below implies

$$
\begin{aligned}
& e_{\chi} \sum_{a=1}^{m_{0}} \zeta_{m_{0}}^{a} \frac{B_{k}\left(\frac{a}{m_{0}}\right)}{k} \\
& =e_{\chi} \sum_{d \mid m_{0}} \sum_{\substack{a^{\prime}=1 \\
\left(a^{\prime}, d\right)=1}}^{d} \zeta_{d}^{a^{\prime}} \frac{B_{k}\left(\frac{a^{\prime}}{d}\right)}{k} \\
& =\sum_{f_{\chi}|d| m_{0}} \sum_{\substack{a^{\prime}=1 \\
\left(a^{\prime}, d\right)=1}}^{d} \frac{\phi\left(f_{\chi}\right)}{\phi(d)} \mu\left(\frac{d}{f_{\chi}}\right) \chi^{-1}\left(\frac{d}{f_{\chi}}\right) \chi\left(a^{\prime}\right) e_{\chi} \zeta_{f_{\chi}} \frac{B_{k}\left(\frac{a^{\prime}}{d}\right)}{k} \\
& =\sum_{f_{\chi}|d| m_{0}} \frac{\phi\left(f_{\chi}\right)}{\phi(d)} \mu\left(\frac{d}{f_{\chi}}\right) \chi^{-1}\left(\frac{d}{f_{\chi}}\right)\left(\sum_{\substack{a^{\prime}=1 \\
\left(a^{\prime}, d\right)=1}}^{d} \chi\left(a^{\prime}\right) \frac{B_{k}\left(\frac{a^{\prime}}{d}\right)}{k}\right) e_{\chi} \zeta_{f_{\chi}} \\
& =\left(\sum_{f_{\chi}|d| m_{0}} \frac{\phi\left(f_{\chi}\right)}{\phi(d)} \mu\left(\frac{d}{f_{\chi}}\right) \chi^{-1}\left(\frac{d}{f_{\chi}}\right) d^{1-k} \prod_{\substack{p \mid d \\
p \nmid f_{\chi}}}\left(1-\chi(p) p^{k-1}\right)\right)(-L(\chi, 1-k)) e_{\chi} \zeta_{f_{\chi}} .
\end{aligned}
$$

Now note that in this last sum only those $d$ contribute for which $d / f_{\chi}$ is both squarefree and prime to $f_{\chi}$. Hence this last expression becomes

$$
\begin{aligned}
& =\prod_{\substack{p \mid m_{0} \\
p \nmid f_{\chi}}}\left(1-\frac{\chi^{-1}(p)}{\phi(p)}\left(p^{1-k}-\chi(p)\right)\right)\left(-f_{\chi}^{1-k} L(\chi, 1-k)\right) e_{\chi} \zeta_{f_{\chi}} \\
& =\prod_{\substack{p \mid m_{0} \\
p \nmid f_{\chi}}}\left(\frac{p-\chi^{-1}(p) p^{1-k}}{p-1}\right)\left(-f_{\chi}^{1-k} L(\chi, 1-k)\right) e_{\chi} \zeta_{f_{\chi}} .
\end{aligned}
$$

On the other hand, using Lemma 1.1 again, we have

$$
\begin{aligned}
e_{\chi} \xi_{m_{0}}=\sum_{m_{1}|d| m_{0}} e_{\chi} \zeta_{d} & =\sum_{m_{1}|d| m_{0}} \frac{\phi\left(f_{\chi}\right)}{\phi(d)} \mu\left(\frac{d}{f_{\chi}}\right) \chi^{-1}\left(\frac{d}{f_{\chi}}\right) e_{\chi} \zeta_{f_{\chi}} \\
& =\prod_{\substack{p \mid m_{0} \\
p \nmid f_{\chi}}} \frac{\mu(p) \chi^{-1}(p)}{\phi(p)} e_{\chi} \zeta_{f_{\chi}}
\end{aligned}
$$


since the only contributing summand is for $d / f_{\chi}=\prod_{\substack{p \mid m_{0} \\ p \nmid f_{\chi}}} p$. Combining (18) and (19), we find

$$
e_{\chi}\left(D^{k} g\right)(0)=\left(1-\chi(l) l^{k-1}\right) \frac{1}{m_{0}} \prod_{\substack{p \mid m_{0} \\ p \nmid f_{\chi}}}\left(p^{1-k}-\chi(p) p\right)\left(-f_{\chi}^{1-k} L(\chi, 1-k)\right) e_{\chi} \xi_{m_{0}}
$$

which together with (17) implies

$$
\chi \chi_{\text {cyclo }}^{k}(\lambda)=-\frac{1}{m_{0}} f_{\chi}^{1-k}\left(\prod_{\substack{p \mid m_{0} \\ p \nmid f_{\chi}}}\left(1-\chi(p) p^{k}\right) p^{1-k}\right)\left(1-\chi(l) l^{k-1}\right) L(\chi, 1-k)
$$

in $\mathbb{Z}_{l}(\chi)$. On the other hand, by (4) combined with the Euler system relations [9], 5.16, for the elements $\theta_{d}$ we have

$$
\begin{aligned}
\chi \chi_{\text {cyclo }}^{k}\left(\kappa^{\#} \theta_{m_{0}}\right) & =\chi \chi_{\text {cyclo }}^{k}\left(\kappa^{\#} \prod_{\substack{p \mid m_{0} \\
p \nmid f_{\chi}}}\left(1-\mathrm{Fr}_{p}^{-1}\right) \theta_{f_{\chi}}\right) \\
& =\prod_{\substack{p \mid m_{0} \\
p \nmid f_{\chi}}}\left(1-\chi(p) p^{k-1}\right)\left(1-\chi(l) l^{k-1}\right) L(\chi, 1-k) .
\end{aligned}
$$

Hence

$$
\chi \chi_{\text {cyclo }}^{k}(\lambda)=-\frac{1}{m_{0}} f_{\chi}^{1-k}\left(\prod_{\substack{p \mid m_{0} \\ p \chi f_{\chi}}} \frac{1-\chi(p) p^{k}}{1-\chi(p) p^{k-1}} p^{1-k}\right) \chi \chi_{\text {cyclo }}^{k}\left(\kappa^{\#} \theta_{m_{0}}\right) .
$$

The definition of $v_{p}$ for $p \mid m_{0}$ ensures that

$$
\chi \chi_{\text {cyclo }}^{k}\left(\prod_{p \mid m_{0}} v_{p}\right)=f_{\chi}^{1-k} \prod_{\substack{p \mid m_{0} \\ p \nmid f_{\chi}}} p^{1-k}
$$

and the definition of $e_{p}$ ensures that

$$
\chi \chi_{\text {cyclo }}^{k}\left(e_{p}\left(1-\mathrm{Fr}_{p}\right)\right)=1-\chi(p) p^{k}
$$

Since $\Lambda$ is reduced and the kernels of characters of the form $\chi \chi_{\text {cyclo }}^{k}$ are dense in $\operatorname{Spec}(\Lambda)$, we conclude

$$
\lambda=-\frac{1}{m_{0}} \prod_{p \mid m_{0}}\left(\frac{e_{p}\left(1-\mathrm{Fr}_{p}\right)}{e_{p}\left(1-\frac{\mathrm{Fr}_{p}}{p}\right)} v_{p}\right) \kappa^{\#}\left(\theta_{m_{0}}\right)
$$

which finishes the proof of Proposition 2.2 for $m_{0}>1$. For $m_{0}=1$, the norm compatible system $\eta_{1}$ does not lie in $U_{\mathrm{loc}}^{\infty}$ but only in the middle term of $(7)$, and $\kappa^{\#}\left(\theta_{1}\right)$ only lies 
in $\frac{1}{\gamma-1} \Lambda$, which is why we have to multiply with $\gamma-1$. The Coleman power series of $(\gamma-1) \eta_{1}$ is

$$
f(X)=\frac{(1+X)^{M}-1}{X}
$$

with $M:=\chi_{\text {cyclo }}(\gamma)=1+\ell m_{0}$. If we define $g(X)$ as in $(16)$ then

$$
\begin{aligned}
D^{k} g(X) & =\left(1-\phi l^{k-1}\right) D^{k-1}(1+X) \frac{f^{\prime}(X)}{f(X)} \\
& =\left(1-\phi l^{k-1}\right) D^{k-1} \frac{M(1+X)^{M}}{(1+X)^{M}-1}-\frac{1+X}{X} \\
& =\left(1-\phi l^{k-1}\right)\left(\frac{d}{d z}\right)^{k-1} \frac{M e^{M z}}{e^{M z}-1}-\frac{e^{z}}{e^{z}-1} \\
& =\left(1-\phi l^{k-1}\right)\left(\frac{d}{d z}\right)^{k-1} \sum_{n=1}^{\infty} \frac{B_{n}(1)}{n}\left(M^{n}-1\right) \frac{z^{n-1}}{(n-1) !}
\end{aligned}
$$

and

$$
\begin{aligned}
\left(D^{k} g\right)(0)=\left(1-l^{k-1}\right) \frac{B_{k}(1)}{k}\left(M^{k}-1\right) & =-\left(M^{k}-1\right)\left(1-l^{k-1}\right) \zeta(1-k) \\
& =\chi_{\text {cyclo }}^{k}\left(-(\gamma-1) \kappa^{\#}\left(\theta_{1}\right)\right) .
\end{aligned}
$$

This finishes the proof for $m_{0}=1$.

Lemma 2.3. For any integer $d$ and Dirichlet character $\chi$ of conductor $f_{\chi} \mid d$ one has

$$
\sum_{\substack{a^{\prime}=1 \\\left(a^{\prime}, d\right)=1}}^{d} \chi\left(a^{\prime}\right) \frac{B_{k}\left(\frac{a^{\prime}}{d}\right)}{k}=-d^{1-k} \prod_{\substack{p \mid d \\ p \nmid f_{\chi}}}\left(1-\chi(p) p^{k-1}\right) L(\chi, 1-k) .
$$

Proof. For $d=f_{\chi}$ this is the well-known formula for Dirichlet $L$-values in terms of Bernoulli polynomials [19], Theorem 4.2, and the general case is proved by induction on the number of primes dividing $d / f_{\chi}$. The distribution relations for the Bernoulli polynomials imply

$$
\begin{aligned}
\sum_{\substack{a=1 \\
(a, d)=1}}^{d p} \chi(a) B_{k}\left(\frac{a}{d p}\right) & =\sum_{\substack{b=1 \\
(b, d)=1}}^{d} \chi(b) \sum_{n=0}^{p-1} B_{k}\left(\frac{b+n d}{d p}\right) \\
& =\sum_{\substack{b=1 \\
(b, d)=1}}^{d} \chi(b) p^{1-k} B_{k}\left(\frac{b}{d}\right)
\end{aligned}
$$


whereas on the other hand

$$
\sum_{\substack{a=1 \\(a, d)=1}}^{d p} \chi(a) B_{k}\left(\frac{a}{d p}\right)=\sum_{\substack{a=1 \\(a, d p)=1}}^{d p} \chi(a) B_{k}\left(\frac{a}{d p}\right)+\sum_{\substack{b=1 \\(b, d)=1}}^{d} \chi(b p) B_{k}\left(\frac{b}{d}\right)
$$

with the convention $\chi(p)=0$ if $p \mid d$. Hence

$$
\sum_{\substack{a=1 \\(a, d p)=1}}^{d p} \chi(a) B_{k}\left(\frac{a}{d p}\right)=\left(p^{1-k}-\chi(p)\right) \sum_{\substack{b=1 \\(b, d)=1}}^{d} \chi(b) B_{k}\left(\frac{b}{d}\right)
$$

We now discuss briefly in what sense Proposition 2.2 can be regarded as a functional equation of the $l$-adic $L$-function $\mathscr{L}$. Artin-Verdier duality gives an exact triangle of perfect complexes of $\Lambda$-modules

$$
R \Gamma_{c}\left(\mathbb{Z}\left[\frac{1}{m_{0} l}\right], T_{l}^{\infty}\right) \rightarrow R \Gamma_{c}\left(\mathbb{Z}\left[\frac{1}{m_{0} l}\right], T_{l}^{\infty, *}(1)\right)^{*}[-3] \rightarrow C\left(T_{l}^{\infty}\right) \rightarrow
$$

where

$$
C\left(T_{l}^{\infty}\right) \cong \bigoplus_{p \mid m_{0} l} R \Gamma\left(\mathbb{Q}_{p}, T_{l}^{\infty}\right) \oplus C\left(\mathbb{R}, T_{l}^{\infty}\right)
$$

and $C\left(\mathbb{R}, T_{l}^{\infty}\right)$ is quasi-isomorphic to a complex

$$
T_{l}^{\infty} \stackrel{1-\phi_{\infty}}{\longrightarrow} T_{l}^{\infty} \stackrel{1+\phi_{\infty}}{\longrightarrow} T_{l}^{\infty}
$$

with $\phi_{\infty} \in \operatorname{Gal}(\overline{\mathbb{Q}} / \mathbb{Q})$ denoting any complex conjugation. This is shown by adapting the arguments in the proof of [6], Proposition 7.2, replacing the projective $\mathfrak{A}_{l}$-module $T_{l}$ by the projective $\Lambda$-module $T_{l}^{\infty}$. The isomorphism of $\operatorname{Gal}(\overline{\mathbb{Q}} / \mathbb{Q})$-modules

$$
T_{l}^{\infty, *}(1) \otimes_{\Lambda, \kappa^{*}} \Lambda \cong T_{l}^{\infty}
$$

induces an isomorphism

$$
\text { (21) } \Delta^{\infty, *}[-3] \otimes_{\Lambda, \kappa^{*}} \Lambda=R \Gamma_{c}\left(\mathbb{Z}\left[\frac{1}{m_{0} l}\right], T_{l}^{\infty, *}(1)\right) \otimes_{\Lambda, \kappa^{*}} \Lambda \cong R \Gamma_{c}\left(\mathbb{Z}\left[\frac{1}{m_{0} l}\right], T_{l}^{\infty}\right) .
$$

Hence the exact triangle (20) can be rewritten

$$
\Delta^{\infty, *}[-3] \otimes_{\Lambda, \kappa^{\#}} \Lambda \rightarrow \Delta^{\infty} \rightarrow C\left(T_{l}^{\infty}\right) \rightarrow
$$

For brevity we now set $Q:=Q(\Lambda)$ and denote the scalar extension to $Q(\Lambda)$ by a subscript $Q$. 
Lemma 2.4. The complex $C\left(\mathbb{R}, T_{l}^{\infty}\right)_{Q}$ has cohomology

$$
H^{i} C\left(\mathbb{R}, T_{l}^{\infty}\right)_{Q}= \begin{cases}\left(T_{l, Q}^{\infty}\right)^{\phi_{\infty}=1} \cong Y_{\{v \mid \infty\}, Q}^{\infty} \otimes_{Q, \kappa^{*}} Q \cong Y_{\left\{v \mid l m_{0} \infty\right\}, Q}^{\infty} \otimes_{Q, \kappa^{\#}} Q, & i=0 \\ \left(T_{l, Q}^{\infty}\right)^{\phi_{\infty}=-1}=Y_{\{v \mid \infty\}, Q}^{\infty} \cong Y_{\left\{v \mid l m_{0} \infty\right\}, Q}^{\infty}, & i=2 \\ 0, & i \neq 0,2\end{cases}
$$

and

$$
\operatorname{Det}_{\Lambda} C\left(\mathbb{R}, T_{l}^{\infty}\right)=\Lambda \cdot\left(2 \sigma+\frac{1}{2} \sigma^{\prime}\right) \subset \operatorname{Det}_{Q} C\left(\mathbb{R}, T_{l}^{\infty}\right)_{Q}
$$

where $\sigma^{\prime}=\sigma \otimes 1$. Note here that $e_{\psi} \sigma^{\prime}=0\left(\right.$ resp. $\left.e_{\psi} \sigma=0\right)$ is $\psi$ is even (resp. odd).

Proof. Note that there is an isomorphism of $\mathbb{Z}_{l}\left[G_{m_{0} l^{n}}\right]$-modules

$$
H^{0}\left(\operatorname{Spec}\left(L_{m_{0} l^{n}} \otimes_{\mathbb{Q}} \overline{\mathbb{Q}}\right), \mathbb{Z}_{l}(1)\right) \cong \bigoplus_{\tau} \mathbb{Z}_{l}
$$

where the sum is over the set of embeddings of $L_{m_{0} l^{n}}$ into $\mathbb{C}$ and so we may naturally view $Y_{\{v \mid \infty\}}\left(L_{m_{0} l^{n}}\right)$ as a quotient of $H^{0}\left(\operatorname{Spec}\left(L_{m_{0} l^{n}} \otimes_{\mathbb{Q}} \overline{\mathbb{Q}}\right), \mathbb{Z}_{l}(1)\right)$ by the action of $c-1$, where $c \in \Lambda$ is the complex conjugation. Passing to the inverse limit over $n$ we find that

$$
H^{2} C\left(\mathbb{R}, T_{l}^{\infty}\right)=T_{l}^{\infty} /\left(\phi_{\infty}+1\right) T_{l}^{\infty}=T_{l}^{\infty} /(c-1) T_{l}^{\infty}=Y_{\{v \mid \infty\}}^{\infty}
$$

since $\phi_{\infty}$ acts on $T_{l}^{\infty}$ via $-c$. Moreover,

$$
H^{0} C\left(\mathbb{R}, T_{l}^{\infty}\right)=\left(T_{l}^{\infty}\right)^{\phi_{\infty}=1}=(c-1) T_{l}^{\infty} \cong Y_{\{v \mid \infty\}}^{\infty} \otimes_{\Lambda, \kappa^{*}} \Lambda .
$$

After localizing at $Q$, we have

$$
Y_{\{v \mid \infty\}, Q}^{\infty} \cong Y_{\left\{v \mid m_{0} l \infty\right\}, Q}^{\infty}
$$

since $Y_{\left\{v \mid m_{0} l\right\}}^{\infty}$ is finite free over $\mathbb{Z}_{l}$ and $C\left(\mathbb{R}, T_{l}^{\infty}\right)_{Q}$ is isomorphic to the complex of $Q$-modules

$$
T_{l, Q}^{\infty,+} \oplus T_{l, Q}^{\infty,-} \stackrel{(2,0)}{\longrightarrow} T_{l, Q}^{\infty,+} \oplus T_{l, Q}^{\infty,-} \stackrel{(0,2)}{\longrightarrow} T_{l, Q}^{\infty,+} \oplus T_{l, Q}^{\infty,-},
$$

where \pm denotes the \pm -eigenspaces for $c$. The determination of $\operatorname{Det}_{\Lambda} C\left(\mathbb{R}, T_{l}^{\infty}\right)$ inside $\operatorname{Det}_{Q} C\left(\mathbb{R}, T_{l}^{\infty}\right)_{Q}$ is then an easy explicit computation (see also the very end of the proof of [6], Proposition 7.2).

Lemma 2.5. For any prime $p \neq l$, the complex $R \Gamma\left(\mathbb{Q}_{p}, T_{l}^{\infty}\right)_{Q}$ is acyclic and one has

$$
\operatorname{Det}_{\Lambda} R \Gamma\left(\mathbb{Q}_{p}, T_{l}^{\infty}\right)=\frac{e_{p}\left(1-\frac{\mathrm{Fr}_{p}}{p}\right)}{e_{p}\left(1-\operatorname{Fr}_{p}\right)} \Lambda \subset Q \cong \operatorname{Det}_{Q} R \Gamma\left(\mathbb{Q}_{p}, T_{l}^{\infty}\right)_{Q} .
$$


Proof. We follow the ideas in the proof of [6], Proposition 7.1. Let $R$ be either a commutative pro- $l$ ring, or a localization of such a ring, and let $C$ be a perfect complex of $R$-modules with a continuous action of $\operatorname{Gal}\left(\overline{\mathbb{F}}_{p} / \mathbb{F}_{p}\right) \cong \hat{\mathbb{Z}} \cdot \phi_{p}^{-1}$. Then the continuous cohomology complex

$$
R \Gamma\left(\mathbb{F}_{p}, C\right) \cong \operatorname{Tot}\left(C \stackrel{1-\phi_{p}^{-1}}{\longrightarrow} C\right)
$$

is a perfect complex of $R$-modules where 'Tot' denotes the total complex of a double complex. The identity map of $C$ induces an isomorphism

$$
\operatorname{id}_{C, \text { triv }}: R \cong \operatorname{Det}_{R} R \Gamma\left(\mathbb{F}_{p}, C\right)
$$

of (graded) invertible $R$-modules. If in addition the complex $R \Gamma\left(\mathbb{F}_{p}, C\right)$ is acyclic, i.e. the differential $d_{C}=1-\phi_{p}^{-1}$ is a quasi-isomorphism, then there is a second isomorphism

$$
d_{C, \text { triv }}: R \cong \operatorname{Det}_{R} R \Gamma\left(\mathbb{F}_{p}, C\right)
$$

arising from acyclicity. Both isomorphisms are functorial for exact triangles in the variable $C$ and commute with scalar extension. They are related by the formula [4], Lemma 1,

$$
\operatorname{id}_{C, \text { triv }}=\left[1-\phi_{p}^{-1}\right] d_{C, \text { triv }}
$$

where $\left[1-\phi_{p}^{-1}\right]$ denotes the class in $K_{1}(R)=R^{\times}$.

We apply these remarks to $R=Q$ and $C=R \Gamma\left(I_{p}, T_{l, Q}^{\infty}\right)$, where $I_{p}$ temporarily denotes the inertia subgroup of $\operatorname{Gal}\left(\overline{\mathbb{Q}}_{p} / \mathbb{Q}_{p}\right)$. Then

$$
R \Gamma\left(\mathbb{F}_{p}, R \Gamma\left(I_{p}, T_{l, Q}^{\infty}\right)\right) \cong R \Gamma\left(\mathbb{Q}_{p}, T_{l, Q}^{\infty}\right) \cong R \Gamma\left(\mathbb{Q}_{p}, T_{l}^{\infty}\right)_{Q}
$$

and there is an exact triangle of perfect complexes of $Q$-modules

$$
H^{0}\left(I_{p}, T_{l, Q}^{\infty}\right)[0] \rightarrow R \Gamma\left(I_{p}, T_{l, Q}^{\infty}\right) \rightarrow H^{1}\left(I_{p}, T_{l, Q}^{\infty}\right)[-1]
$$

On the $Q$-module $H^{0}\left(I_{p}, T_{l, Q}^{\infty}\right)=\left(T_{l, Q}^{\infty}\right)^{I_{p}} \quad\left(\right.$ resp. $\left.H^{1}\left(I_{p}, T_{l, Q}^{\infty}\right) \cong\left(T_{l, Q}^{\infty}\right)_{I_{p}}(-1)\right)$ the map $1-\phi_{p}^{-1}$ acts by the element $e_{p}\left(1-\operatorname{Fr}_{p} / p\right)\left(\right.$ resp. $\left.e_{p}\left(1-\operatorname{Fr}_{p}\right)\right)$ of $Q^{\times}$. By the discussion above, we have

$$
\begin{aligned}
\operatorname{id}_{R \Gamma\left(I_{p}, T_{l}^{\infty}\right), \text { triv }} \otimes_{\Lambda} Q & =\mathrm{id}_{R \Gamma\left(I_{p}, T_{l, Q}^{\infty}\right), \text { triv }} \\
& =\mathrm{id}_{T_{l, Q}^{\infty}, I_{p}, \text { triv }} \otimes \operatorname{id}_{\left(T_{l, Q}^{\infty}\right)_{I_{p}}(-1), \text { triv }}^{-1} \\
& =e_{p}\left(1-\operatorname{Fr}_{p} / p\right) d_{T_{l, Q}^{\infty, I_{p}}, \text { triv }} \otimes e_{p}\left(1-\operatorname{Fr}_{p}\right)^{-1} d_{\left(T_{l, Q}^{-\infty}\right)_{I_{p}}(-1), \text { triv }} \\
& =\frac{e_{p}\left(1-\mathrm{Fr}_{p} / p\right)}{e_{p}\left(1-\mathrm{Fr}_{p}\right)} d_{R \Gamma\left(I_{p}, T_{l, Q}^{\infty}\right), \text { triv }} .
\end{aligned}
$$


Together with (23) this means that the lattice $\frac{e_{p}\left(1-\operatorname{Fr}_{p} / p\right)}{e_{p}\left(1-\operatorname{Fr}_{p}\right)} \Lambda \subseteq Q$ is mapped to

$$
\operatorname{Det}_{\Lambda} R \Gamma\left(\mathbb{Q}_{p}, T_{l}^{\infty}\right) \subset \operatorname{Det}_{Q} R \Gamma\left(\mathbb{Q}_{p}, T_{l, Q}^{\infty}\right)
$$

under $d_{R \Gamma\left(I_{p}, T_{l, Q}^{\infty}\right), \text { triv }}$, i.e. the statement of the lemma.

The following statement might be regarded as a functional equation of $\mathscr{L}$.

Proposition 2.6. Under the isomorphism

$$
\operatorname{Det}_{Q} \Delta_{Q}^{\infty} \cong\left(\operatorname{Det}_{Q} \Delta_{Q}^{\infty} \otimes_{Q, \kappa^{*}} Q\right) \otimes_{Q} \bigotimes_{p \mid m_{0} l} \operatorname{Det}_{Q} R \Gamma\left(\mathbb{Q}_{p}, T_{l}^{\infty}\right)_{Q} \otimes \operatorname{Det}_{Q} C\left(\mathbb{R}, T_{l}^{\infty}\right)_{Q}
$$

induced by the exact triangle $(22)_{Q}$, we have

$$
\mathscr{L}=\kappa^{\#}(\mathscr{L}) \otimes-m_{0} \prod_{p \mid m_{0}}\left(\frac{e_{p}\left(1-\mathrm{Fr}_{p} / p\right)}{e_{p}\left(1-\mathrm{Fr}_{p}\right)} v_{p}^{-1}\right) \cdot\left(\beta_{m_{0}}^{\infty}\right)^{-1} \otimes\left(2 \sigma+\frac{1}{2} \sigma^{\prime}\right)
$$

Proof. The long exact cohomology sequence of the triangle $(22)_{Q}$ reads

$$
\begin{aligned}
0 & \rightarrow H^{0} C\left(\mathbb{R}, T_{l}^{\infty}\right)_{Q} \rightarrow H^{2}\left(\Delta^{\infty}\right)_{Q}^{*} \otimes_{Q, \kappa^{*}} Q \rightarrow H^{1}\left(\Delta_{\infty}\right)_{Q} \rightarrow H^{1}\left(\mathbb{Q}_{l}, T_{l}^{\infty}\right)_{Q} \\
& \rightarrow H^{1}\left(\Delta^{\infty}\right)_{Q}^{*} \otimes_{Q, \kappa^{*}} Q \rightarrow H^{2}\left(\Delta^{\infty}\right)_{Q} \rightarrow H^{2} C\left(\mathbb{R}, T_{l}^{\infty}\right)_{Q} \rightarrow 0
\end{aligned}
$$

or more concretely, given the computation of $H^{i}(\Delta)\left(\right.$ resp. $\left.H^{i}\left(\mathbb{Q}_{l}, T_{l}^{\infty}\right)\right)$ in Section 1.2 (resp. (6))

$$
\begin{aligned}
0 & \rightarrow Y_{\{v \mid \infty\}, Q}^{\infty} \otimes_{Q, \kappa^{*}} Q \rightarrow Y_{\{v \mid \infty\}, Q}^{\infty, *} \otimes_{Q, \kappa^{\#}} Q \rightarrow U_{\left\{v \mid l m_{0}\right\}, Q}^{\infty} \rightarrow U_{\mathrm{loc}, Q}^{\infty} \\
& \rightarrow U_{\left\{v \mid l m_{0}\right\}, Q}^{\infty, *} \otimes_{Q, \kappa^{\#}} Q \rightarrow Y_{\{v \mid \infty\}, Q}^{\infty} \rightarrow Y_{\{v \mid \infty\}, Q}^{\infty} \rightarrow 0 .
\end{aligned}
$$

Noting that $Q=\prod Q(\psi)$ is a product ring (see $\left.(5)\right)$ this sequence becomes for even $\psi$

$$
0 \rightarrow 0 \rightarrow 0 \rightarrow U_{\left\{v \mid l m_{0}\right\}, Q(\psi)}^{\infty} \rightarrow U_{\mathrm{loc}, Q(\psi)}^{\infty} \rightarrow 0 \rightarrow Y_{\{v \mid \infty\}, Q(\psi)}^{\infty} \rightarrow Y_{\{v \mid \infty\}, Q(\psi)}^{\infty} \rightarrow 0
$$

Taking the bases of the modules in this sequence and using Proposition 2.2 we find

$$
2 \cdot \eta_{m_{0}}^{-1} \otimes \sigma=-m_{0} \prod_{p \mid m_{0}}\left(\frac{e_{p}\left(1-\mathrm{Fr}_{p} / p\right)}{e_{p}\left(1-\mathrm{Fr}_{p}\right)} v_{p}^{-1}\right) \kappa^{\#}\left(\theta_{m_{0}}\right)^{-1} \cdot\left(\beta_{m_{0}}^{\infty}\right)^{-1} \otimes 2 \cdot \sigma
$$

which is the even component of equation (24). For odd $\psi$ the sequence $(25)$ reads

$$
0 \rightarrow Y_{\{v \mid \infty\}, Q\left(\omega \psi^{-1}\right)}^{\infty} \rightarrow Y_{\{v \mid \infty\}, Q\left(\omega \psi^{-1}\right)}^{\infty, *} \rightarrow 0 \rightarrow U_{\mathrm{loc}, Q(\psi)}^{\infty} \rightarrow U_{\left\{v \mid l m_{0}\right\}, Q\left(\omega \psi^{-1}\right)}^{\infty, *} \rightarrow 0 \rightarrow 0 \rightarrow 0
$$

and a similar application of Proposition 2.2 gives the odd component of (24). 
Remark. Note that the element

$$
-m_{0} \prod_{p \mid m_{0}}\left(\frac{e_{p}\left(1-\frac{\mathrm{Fr}_{p}}{p}\right)}{e_{p}\left(1-\mathrm{Fr}_{p}\right)} v_{p}^{-1}\right) \cdot\left(\beta_{m_{0}}^{\infty}\right)^{-1} \otimes\left(2 \sigma+\frac{1}{2} \sigma^{\prime}\right)
$$

is a $\Lambda$-basis of

$$
\bigotimes_{p \mid m_{0} l} \operatorname{Det}_{\Lambda} R \Gamma\left(\mathbb{Q}_{p}, T_{l}^{\infty}\right) \otimes_{\Lambda} \operatorname{Det}_{\Lambda} C\left(\mathbb{R}, T_{l}^{\infty}\right)
$$

by Proposition 1.4, Lemmas 2.4 and 2.5. Proposition 2.6 then implies that

$$
\mathscr{L} \otimes\left(\kappa^{\#}(\mathscr{L})\right)^{-1}
$$

is a $\Lambda$-basis of

$$
\operatorname{Det}_{\Lambda} \Delta^{\infty} \otimes_{\Lambda}\left(\operatorname{Det}_{\Lambda} \Delta^{\infty} \otimes_{\Lambda, \kappa^{\#}} \Lambda\right)^{-1}
$$

which is also a (rather weak) consequence of Theorem 1.2.

\section{The proof of Theorem 1.2 for $l=2$}

3.1. Recollections from [9]. In this section, we continue the proof of Theorem 1.2 for $l=2$ where we have left it off in [9]. By [9], Lemma 5.3, it suffices to show that

$$
\Lambda_{\mathfrak{q}} \cdot \mathscr{L}=\operatorname{Det}_{\Lambda_{\mathfrak{q}}} \Delta_{\mathfrak{q}}^{\infty}
$$

for all height one primes $\mathfrak{q}$ of $\Lambda$, and primes $\mathfrak{q}$ not containing $l=2$ have already been dealt with in [9].

In the following $l$ will always denote 2 and $\mathfrak{q}$ a height one prime of $\Lambda$ containing 2 . Such primes are in bijection with $\mathbb{Q}_{l}$-rational characters $\psi_{\mathfrak{q}}$ of $G_{m_{0} l^{\infty}}^{\text {tor }} \cong G_{m_{0} \ell}$ of order prime to $l$. We let $c \in \Lambda$ be the complex conjugation. Then it was shown in [9], p. 95, that $\Delta_{\mathfrak{q}}^{\infty}$ is quasi-isomorphic to a complex

$$
\Delta_{\mathfrak{q}}^{\infty, 1} \stackrel{\partial^{\prime}}{\rightarrow} \Delta_{\mathfrak{q}}^{\infty, 2}
$$

where both $\Lambda_{\mathfrak{q}}$-modules $\Delta_{\mathfrak{q}}^{\infty, i}$ are free of rank one and the image of the differential $\partial^{\prime}$ is $(c-1) \Delta_{\mathfrak{q}}^{\infty, 2}$. Following [9], we pick $\Lambda_{\mathfrak{q}}$-bases $\gamma_{i}$ of $\Delta_{\mathfrak{q}}^{\infty, i}$, so that

$$
\gamma_{2} \mapsto \sigma \in H^{2}\left(\Delta_{\mathfrak{q}}^{\infty}\right), \quad(c+1) \gamma_{1}=\eta_{m_{0}} \in H^{1}\left(\Delta_{\mathfrak{q}}^{\infty}\right),
$$

and define $\alpha \in(c-1) \Lambda_{\mathfrak{q}}^{\times}$by

$$
\partial^{\prime}\left(\gamma_{1}\right)=\alpha \gamma_{2}
$$


These conditions determine $\gamma_{1}, \gamma_{2}$ and therefore $\alpha$ up to factors in $1+(c-1) \Lambda_{\mathfrak{q}}$. The class of $\alpha$ in

$$
(c-1) \Lambda_{\mathfrak{q}} /(c-1)^{2} \Lambda_{\mathfrak{q}}=(c-1) \Lambda_{\mathfrak{q}} / 2(c-1) \Lambda_{\mathfrak{q}} \cong \Lambda_{\mathfrak{q}} /(2, c+1)
$$

is therefore independent of all choices and contains the same information as the Yoneda two-extension class in

$$
\operatorname{Ext}_{\Lambda_{\mathfrak{q}}}^{2}\left(H^{2}\left(\Delta_{\mathfrak{q}}^{\infty}\right), H^{1}\left(\Delta_{\mathfrak{q}}^{\infty}\right)\right) \cong \Lambda_{\mathfrak{q}} /(2, c+1)
$$

which is represented by the complex $\Delta_{\mathfrak{q}}^{\infty}$.

We have

$$
\operatorname{Det}_{\Lambda_{\mathfrak{q}}} \Delta_{\mathfrak{q}}^{\infty}=\Lambda_{\mathfrak{q}} \cdot \gamma_{1}^{-1} \otimes \gamma_{2}=\Lambda_{\mathfrak{q}} \cdot u \cdot \mathscr{L}
$$

for some $u \in Q(\Lambda)$ and (26) becomes the statement that $u \in \Lambda_{\mathfrak{q}}^{\times}$. We may compute $u=\left(u_{\psi}\right)$ over $Q(\Lambda) \cong \prod_{\psi} Q(\psi)$, a product of fields indexed by the $\mathbb{Q}_{l}$-rational characters $G_{m_{0} \ell}$. For even $\psi$ (with prime-to-l part $\psi_{\mathfrak{q}}$ ) we have as in [9]

$$
e_{\psi}\left(\gamma_{1}^{-1} \otimes \gamma_{2}\right)=e_{\psi}\left(\left(\frac{1}{2} \eta_{m_{0}}\right)^{-1} \otimes \sigma\right)=e_{\psi} \mathscr{L}
$$

and hence $u_{\psi}=1$. For odd $\psi$ the canonical basis of $\Delta^{\infty} \otimes_{\Lambda} Q(\psi)$ arising from the acyclicity of this complex is $\gamma_{1}^{-1} \otimes \alpha \gamma_{2}$ and hence

$$
e_{\psi}\left(\gamma_{1}^{-1} \otimes \gamma_{2}\right)=e_{\psi}\left(\frac{1}{\alpha} \cdot \gamma_{1}^{-1} \otimes \alpha \gamma_{2}\right)=e_{\psi}\left(\frac{\theta_{m_{0}}}{\alpha} \cdot \mathscr{L}\right)
$$

so that $u_{\psi}=e_{\psi}\left(\frac{\theta_{m_{0}}}{\alpha}\right)$. In [9] we were erroneously assuming that $\alpha=1-c$ and that the resulting $u$ lies in $\Lambda_{\mathfrak{q}}^{\times}$.

Lemma 3.1. Set $l=2$, let $\mathfrak{q}$ be a height 1 prime of $\Lambda$ containing 2 and let $\alpha$ be defined by (27). If we have

$$
\alpha=\theta_{m_{0}}(1+(c-1) x+(c+1) y)
$$

for some $x, y \in \Lambda_{\mathfrak{q}}$ then (26), and a forteriori Theorem 1.2, hold.

Proof. Indeed, we then have $u_{\psi}=e_{\psi}(1+(c-1) x+(c+1) y)=e_{\psi}(1+(c-1) x)$ for odd $\psi$. Since $u_{\psi}=1$ for even $\psi$ we have $u_{\psi}=e_{\psi}(1+(c-1) x)$ for all $\psi$. Hence $u=1+(c-1) x \in \Lambda_{\mathfrak{q}}^{\times}$. 
The complex $R \Gamma_{c}\left(\mathbb{Z}\left[\frac{1}{m_{0} l}\right], T_{l}^{\infty}\right)$ turns out to be more amenable to direct analysis than $\Delta^{\infty}$ (recall that the two are related by (21)). After describing an explicit representative of this complex and a basis of its determinant we shall verify that $\alpha$ has the shape (28) in Section 3.4.

3.2. Explicit construction of $R \Gamma_{c}\left(\mathbb{Z}\left[\frac{1}{m_{0} l}\right], T_{l}^{\infty}\right)$. For a profinite group $G$ and a continuous $G$-module $M$, we denote by $R \Gamma(G, M)\left(\operatorname{resp} . Z^{i}(G, M)\right)$ the complex of continuous cochains (resp. the group of continuous cocycles of degree $i$ ). We let $\Sigma$ be the set of primes dividing $m_{0} l, L_{\Sigma}$ the maximal algebraic extension of $\mathbb{Q}$ unramified outside $\Sigma$ and set $G_{\Sigma}=\operatorname{Gal}\left(L_{\Sigma} / \mathbb{Q}\right)$. There is a natural isomorphism [16], Proposition 2.9,

$$
R \Gamma\left(\mathbb{Z}\left[\frac{1}{m_{0} l}\right], T_{l}^{\infty}\right) \cong R \Gamma\left(G_{\Sigma}, T_{l}^{\infty}\right)
$$

and we shall also identify $R \Gamma\left(\mathbb{Q}_{p}, T_{l}^{\infty}\right)$ and $R \Gamma\left(\mathbb{R}, T_{l}^{\infty}\right)$ with the complex of continuous cochains of the respective Galois group. By definition

$$
R \Gamma_{c}\left(\mathbb{Z}\left[\frac{1}{m_{0} l}\right], T_{l}^{\infty}\right)=\operatorname{Cone}\left(R \Gamma\left(G_{\Sigma}, T_{l}^{\infty}\right) \rightarrow \bigoplus_{p \mid m_{0} l \infty} R \Gamma\left(\mathbb{Q}_{p}, T_{l}^{\infty}\right)\right)[-1]
$$

We denote by

$$
d:=d^{0}: T_{l}^{\infty} \stackrel{x \mapsto(g \mapsto(g-1) x)}{\longrightarrow} Z^{1}\left(G_{\Sigma}, T_{l}^{\infty}\right)=\operatorname{ker}\left(d^{1}\right)
$$

the 0 -th differential in the complex $R \Gamma\left(G_{\Sigma}, T_{l}^{\infty}\right)$ and by $\operatorname{res}_{p}\left(\operatorname{resp} . \overline{\mathrm{res}}_{p}\right)$ the restriction map of cochains (resp. cohomology classes) to a fixed decomposition group at $p$.

Proposition 3.2. (a) The complex $R \Gamma_{c}\left(\mathbb{Z}\left[\frac{1}{m_{0} l}\right], T_{l}^{\infty}\right)_{\mathfrak{q}}$ is naturally quasi-isomorphic to the complex concentrated in degrees 1 and 2

$$
K_{\mathfrak{q}} \stackrel{\partial}{\rightarrow} H^{1}\left(\mathbb{Q}_{l}, T_{l}^{\infty}\right)_{\mathfrak{q}}
$$

where

$$
K:=\left\{z \in Z^{1}\left(G_{\Sigma}, T_{l}^{\infty}\right) \mid \operatorname{res}_{\infty}(z)=0\right\}
$$

and $\partial(z):=\overline{\operatorname{res}}_{l} z$.

(b) The natural map

$$
(c-1) T_{l, \mathfrak{q}}^{\infty}=H^{0}\left(\mathbb{R}, T_{l}^{\infty}\right)_{\mathfrak{q}} \cong H_{c}^{1}\left(\mathbb{Z}\left[\frac{1}{m_{0} l}\right], T_{l}^{\infty}\right)_{\mathfrak{q}} \cong \operatorname{ker}(\partial),
$$


where the second isomorphism arises from the cohomology sequence of (29), is given by $x \mapsto d x$, and the map

$$
H^{1}\left(\mathbb{Q}_{l}, T_{l}^{\infty}\right)_{\mathfrak{q}} \rightarrow \operatorname{coker}(\partial) \cong H_{c}^{2}\left(\mathbb{Z}\left[\frac{1}{m_{0} l}\right], T_{l}^{\infty}\right)_{\mathfrak{q}}
$$

is the one in the cohomology sequence of (29).

(c) The modules $K_{\mathfrak{q}}$ and $H^{1}\left(\mathbb{Q}_{l}, T_{l}^{\infty}\right)_{\mathfrak{q}}$ are both free of rank one over $\Lambda_{\mathfrak{q}}$.

Proof. By Lemma 3.3 below we may ignore the summands for primes $p \neq l, \infty$ in (29) and for $p=l$ (resp. $p=\infty)$ the complex $R \Gamma\left(\mathbb{Q}_{p}, T_{l}^{\infty}\right)_{\mathfrak{q}}$ is concentrated in degree 1 (resp. 0). For $p=l$, this follows from (6) and for $p=\infty$ the complex $R \Gamma\left(\mathbb{R}, T_{l}^{\infty}\right)$ is quasiisomorphic to

$$
T_{l}^{\infty} \stackrel{c+1}{\longrightarrow} T_{l}^{\infty} \stackrel{c-1}{\longrightarrow} T_{l}^{\infty} \stackrel{c+1}{\longrightarrow} \cdots .
$$

Moreover, a computation using Artin-Verdier duality shows that $H^{i}\left(G_{\Sigma}, T_{l}^{\infty}\right)_{\mathfrak{q}}=0$ for $i \geqq 2$ and hence that $R \Gamma_{c}\left(\mathbb{Z}\left[\frac{1}{m_{0} l}\right], T_{l}^{\infty}\right)_{\mathfrak{q}}$ is naturally quasi-isomorphic to

$$
\operatorname{Cone}\left(\tau^{\leqq 1} R \Gamma\left(G_{\Sigma}, T_{l}^{\infty}\right) \rightarrow H^{1}\left(\mathbb{Q}_{l}, T_{l}^{\infty}\right)[-1] \oplus \tau^{\leqq 1} R \Gamma\left(\mathbb{R}, T_{l}^{\infty}\right)\right)_{\mathfrak{q}}[-1]
$$

Written out explicitly this mapping cone is the $\mathfrak{q}$-localization of the complex in the first row of the following commutative diagram:

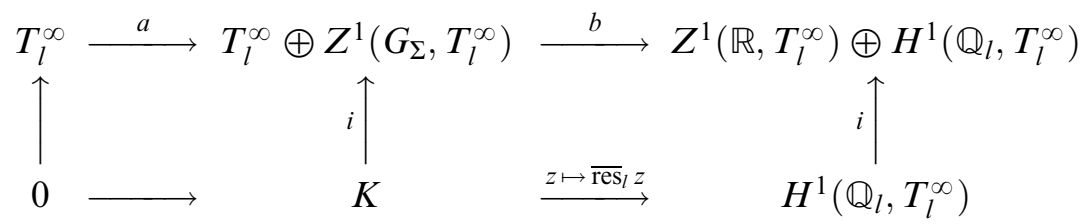

where $a(x)=(x,-d x), b(x, y)=\left(\operatorname{res}_{\infty}(d x+y), \overline{\operatorname{res}}_{l} y\right)$ and $i(z)=(0, z)$. We show that the vertical map of complexes is a quasi-isomorphism. Since $a$ is injective we get a cohomology isomorphism in degree 0 . If $(x, y) \in \operatorname{ker}(b) / \operatorname{im}(a)$ then

$$
(x, y)=(0, d x+y)+(x,-d x) \equiv i(d x+y) \quad \bmod \operatorname{im}(a)
$$

and $d x+y \in \operatorname{ker}(\partial)$ since $\overline{\operatorname{res}}_{l} \circ d=0$. If $z \in \operatorname{ker}(\partial)$ and $i(z)=(0, z)=(x,-d x) \in \operatorname{im}(a)$ then $x=d x=z=0$, hence we get a cohomology isomorphism in degree 1 . Since $T_{l}^{\infty} \stackrel{\operatorname{res}_{\infty} \circ d}{\longrightarrow} Z^{1}\left(\mathbb{R}, T_{l}^{\infty}\right)$ is surjective, for any given $(\xi, \eta) \in Z^{1}\left(\mathbb{R}, T_{l}^{\infty}\right) \oplus H^{1}\left(\mathbb{Q}_{l}, T_{l}^{\infty}\right)$, we find $x \in T_{l}^{\infty}$ so that

$$
(\xi, \eta)=(0, \eta)+\left(\operatorname{res}_{\infty}(d x), 0\right) \equiv i(\eta) \operatorname{modim}(b) .
$$

On the other hand, if $i(\eta)=(0, \eta)=\left(\operatorname{res}_{\infty}(d x+y), \overline{\operatorname{res}}_{l} y\right) \in \operatorname{im}(b)$ then $d x+y \in K$ and $\eta=\overline{\operatorname{res}}_{l}(d x+y) \in \operatorname{im}(\partial)$. Hence we get a cohomology isomorphism in degree 2 and the 

statement (a). The map of complexes $\tau^{\leqq 1} R \Gamma\left(\mathbb{R}, T_{l}^{\infty}\right) \rightarrow R \Gamma_{c}\left(\mathbb{Z}\left[\frac{1}{m_{0} l}\right], T_{l}^{\infty}\right)[1]$ is explicitly
given by

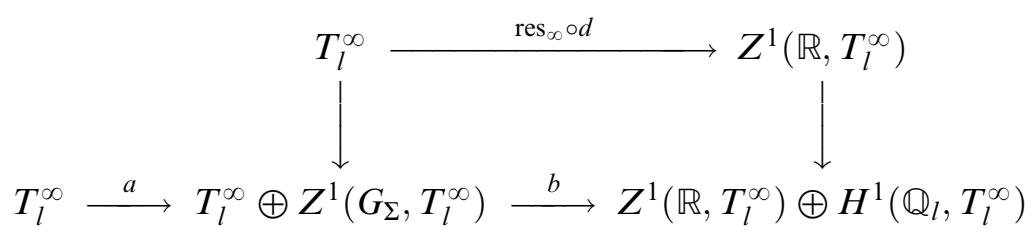

where the vertical maps are the inclusions into the respective first summands. Evaluating a 1-cocycle $f$ on a complex conjugation $\phi_{\infty} \in G_{\Sigma}$ gives an isomorphism

$$
Z^{1}\left(\mathbb{R}, T_{l}^{\infty}\right) \cong T_{l}^{\infty, \phi_{\infty}=-1} \cong T_{l}^{\infty, c=1}=(c+1) T_{l}^{\infty}
$$

and the differential res r $_{\infty} \circ d$ is given by $x \mapsto(c+1) x$. Its kernel is $(c-1) T_{l}^{\infty}$ which maps into $Z^{1}\left(\mathbb{R}, T_{l}^{\infty}\right) \oplus H^{1}\left(\mathbb{Q}_{l}, T_{l}^{\infty}\right)$ via $x \mapsto(x, 0)$. The first part of (b) then follows from (32), and the second part is clear from the mapping cone construction.

The $\Lambda_{\mathfrak{q}}$-module $H^{1}\left(\mathbb{Q}_{l}, T_{l}^{\infty}\right)_{\mathfrak{q}}$ is free of rank one with basis $\beta_{m_{0}}^{\infty}$ by (6), (7), (8) and Lemma 1.3. A diagram chase using (34) shows that the following commutative diagram has exact rows and columns (even before localization at $\mathfrak{q}$ ):

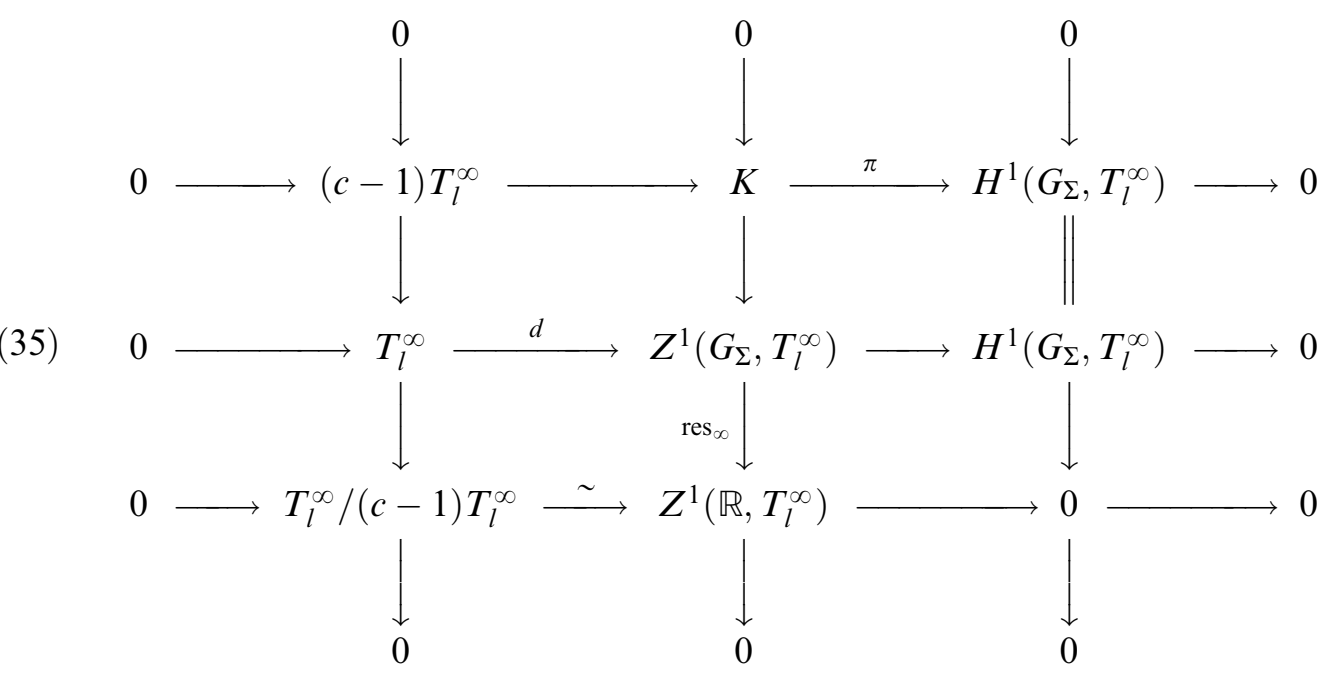

Combining this diagram with the isomorphism [9], p. 94,

$$
H^{1}\left(G_{\Sigma}, T_{l}^{\infty}\right)_{\mathfrak{q}} \cong\left(\lim _{n} \mathcal{O}_{L_{m_{0} l^{n}}}\left[\frac{1}{m_{0} l}\right]^{\times} \otimes_{\mathbb{Z}} \mathbb{Z}_{l}\right)_{\mathfrak{q}} \cong H^{1}\left(\Delta^{\infty}\right)_{\mathfrak{q}}=\left(\Lambda_{\mathfrak{q}} /(c-1)\right) \cdot \eta_{m_{0}}
$$

we find that $K_{\mathfrak{q}}$ is isomorphic to an extension of $\Lambda_{\mathfrak{q}} /(c-1)$ by $\Lambda_{\mathfrak{q}} /(c+1)$. On the other hand, $K_{\mathfrak{q}}$ is of finite projective dimension over $\Lambda_{\mathfrak{q}}$ since $R \Gamma_{c}\left(\mathbb{Z}\left[\frac{1}{m_{0} l}\right], T_{l}^{\infty}\right)_{\mathfrak{q}}$ is perfect. An argument as in [9], equation (5.17), then shows that $K_{\mathfrak{q}}$ must be isomorphic to $\Lambda_{\mathfrak{q}}$.

Lemma 3.3. For $p \neq l, \infty$ the complex $R \Gamma\left(\mathbb{Q}_{p}, T_{l}^{\infty}\right)_{\mathfrak{q}}$ is acyclic. 
Proof. Similarly to (6) the cohomology of $R \Gamma\left(\mathbb{Q}_{p}, T_{l}^{\infty}\right)$ is naturally isomorphic to

$$
H^{i}\left(\mathbb{Q}_{p}, T_{l}^{\infty}\right) \cong \begin{cases}\left(\lim _{n} \mathbb{Q}\left(\zeta_{m_{0} l^{n}}\right)_{p}^{\times} / l^{n}\right) \cong \prod_{v \mid p} \mathbb{Z}_{l}(1), & i=1, \\ \prod_{v \mid p} \mathbb{Z}_{l}, & i=2, \\ 0, & \text { otherwise }\end{cases}
$$

where $\mathbb{Q}\left(\zeta_{m_{0} l^{n}}\right)_{p}:=\mathbb{Q}\left(\zeta_{m_{0} l^{n}}\right) \otimes_{\mathbb{Q}} \mathbb{Q}_{p}$ is a finite product of local fields and the limit is taken with respect to the norm maps. Since the $\mu$-invariant of any $\mathbb{Z} \llbracket \llbracket \gamma-1 \rrbracket$-module which is finite free as a $\mathbb{Z}_{l}$-module vanishes we conclude by [9], Lemma 5.6, that $H^{i}\left(\mathbb{Q}_{p}, T_{l}^{\infty}\right)_{\mathfrak{q}}=0$.

3.3. Description of a basis of $\boldsymbol{K}_{\mathfrak{q}}$. In order to describe elements in $K_{\mathfrak{q}}$ we shall use the computations in the appendix to construct a set-theoretic section $z$ of the map $\pi$ in (35).

Lemma 3.4. Let

$$
u=\left(u_{n}\right) \in U_{\left\{v \mid m_{0} l\right\}}^{\infty}:=\lim _{n} \mathcal{O}_{L_{m_{0} l^{n}}}\left[\frac{1}{m_{0} l}\right]^{\times} \otimes_{\mathbb{Z}} \mathbb{Z}_{l} \cong H^{1}\left(G_{\Sigma}, T_{l}^{\infty}\right)
$$

be a norm compatible system of real units, i.e. so that $(c-1) u=0$. Then if $z \in K$ is any lift (i.e. $\pi(z)=u$ ) and we write

$$
(c-1) z=d\left(\lambda_{\infty} \sigma\right),
$$

then $\lambda_{\infty}=\left(\lambda_{n}\right) \in(c-1) \Lambda$ satisfies

$$
\lambda_{n} \equiv \sum_{a \bmod m_{0} l^{n}} \varepsilon_{n, a} \tau_{a, m_{0} l^{n}}^{-1} \bmod 2
$$

where $\varepsilon_{n, a} \in\{0,1\}$ is defined by $(-1)^{\varepsilon_{n, a}}=\operatorname{sgn}\left(\sigma_{m_{0} l n}\left(u_{n}^{\tau_{a, m_{0} l^{n}}}\right)\right)$. The class of $\lambda_{\infty}$ modulo $2(c-1) \Lambda$ only depends on $u$.

Proof. Since

$$
0=(c+1)(c-1) z=(c+1) d\left(\lambda_{\infty} \sigma\right)=d\left((c+1) \lambda_{\infty} \sigma\right)
$$

and $d$ is injective we have $(c+1) \lambda_{\infty}=0$, i.e. $\lambda_{\infty} \in(c-1) \Lambda$. Changing $z$ to $z+d((c-1) x)$ with $x \in T_{l}^{\infty}$, see diagram (35), will clearly change $\lambda_{\infty}$ by an element in

$$
(c-1)^{2} \Lambda=2(c-1) \Lambda .
$$

We conclude that $u$ determines $\lambda_{\infty}$ modulo $2(c-1) \Lambda$.

In order to prove the congruence for $\lambda_{n} \in \Lambda_{n}:=\mathbb{Z} / l^{n} \mathbb{Z}\left[G_{\left.m_{0}\right|^{n}}\right]$ we apply the discussion in the appendix to $G=\Gamma_{n}, H=H_{n}$ and $M=\mu_{l^{n}}$ where

$$
H_{n}=\operatorname{Gal}\left(F_{m_{0} l^{n}} / L_{m_{0} l^{n}}\right), \quad \Gamma_{n}=\operatorname{Gal}\left(F_{m_{0} l^{n}} / \mathbb{Q}\right)
$$


and $F_{m_{0} l^{n}}$ denotes the maximal abelian extension of $L_{m_{0} l^{n}}$ unramified outside $\Sigma$ and annihilated by $l^{n}$. We denote by $\sigma_{m_{0} l^{n}}: F_{m_{0} l^{n}} \rightarrow \mathbb{C}$ an embedding which extends $\sigma_{m_{0} l^{n}}$ on $L_{m_{0} l^{n}}$ and note that the embedding $\sigma_{m_{0} l^{n}}$ determines a surjection $\operatorname{Gal}(\overline{\mathbb{Q}} / \mathbb{Q}) \rightarrow \Gamma_{n}$ which allows us to view $\phi_{\infty}$ as an element of $\Gamma_{n}$.

We let $S=S_{n}$ be a set of representatives of $H_{n} \backslash \Gamma_{n}$ which not only contains 1 but is also right invariant under $\phi_{\infty}$. This we can achieve by picking a set $\tilde{S} \subset \Gamma_{n}$ which maps to a set of right coset representatives of $\phi_{\infty} \in G / H \cong G_{m_{0} l^{n}}$ and setting $S=\tilde{S} \cup \tilde{S} \phi_{\infty}$. Then we have $h(x)=h\left(x \phi_{\infty}\right)$ and the cocycle $l(f)$ constructed in Lemma 4.1 therefore satisfies

$$
l(f)\left(\phi_{\infty}\right)=0
$$

for any homomorphism $f: H_{n} \rightarrow \mu_{l^{n}}$. We have an isomorphism of free, rank one $\Lambda_{n}$-modules

$$
\begin{aligned}
\operatorname{Coind}_{H_{n}}^{\Gamma_{n}} \mu_{l^{n}} & =\operatorname{Hom}_{\mathbb{Z}\left[H_{n}\right]}\left(\mathbb{Z}\left[\Gamma_{n}\right], \mu_{l^{n}}\right) \cong \operatorname{Hom}_{\mathbb{Z} / l^{n} \mathbb{Z}}\left(\Lambda_{n}, \mu_{l^{n}}\right) \\
& \cong H^{0}\left(\operatorname{Spec}\left(L_{m_{0} l^{n}} \otimes_{\mathbb{Q}} \overline{\mathbb{Q}}\right), \mu_{l^{n}}\right)=\operatorname{Map}\left(\mathscr{T}_{n}, \mu_{l^{n}}\right)=: \mathscr{F}_{n}
\end{aligned}
$$

where $\mathscr{T}_{n}=\operatorname{Hom}_{\text {Rings }}\left(L_{m_{0} l^{n}}, \overline{\mathbb{Q}}\right)$ with its natural action of $G_{m_{0} l^{n}}$ and $\operatorname{Gal}(\overline{\mathbb{Q}} / \mathbb{Q})$ and

$$
\operatorname{Hom}_{\mathbb{Z} / l^{n} \mathbb{Z}}\left(\Lambda_{n}, \mu_{l^{n}}\right) \ni \psi \mapsto \sum_{g \in G_{m_{0}} l^{n}} \hat{\psi}(g) g \cdot \sigma_{m_{0} l^{n}} .
$$

Here $\hat{\psi}(g) \in \mathbb{Z} / l^{n} \mathbb{Z}$ is defined by ${ }^{g^{-1}} \psi(g)=\zeta_{l^{n}}^{\hat{\psi}(g)}$ and we also denote by $\sigma_{m_{0} l^{n}}$ the element of $\mathscr{F}_{n}$ sending $\sigma_{m_{0} l^{n}}$ to $\zeta_{l^{n}}$ and all other $\tau \in \mathscr{T}_{n}$ to 0 . Moreover

$$
\underset{n}{\lim _{n}} H^{1}\left(H_{n}, \mu_{l^{n}}\right) \cong \lim _{n} H^{1}\left(\Gamma_{n}, \mathscr{F}_{n}\right) \cong \underset{n}{\lim _{n}} H^{1}\left(G_{\Sigma}, \mathscr{F}_{n}\right) \cong H^{1}\left(G_{\Sigma}, T_{l}^{\infty}\right)
$$

where the first isomorphism is given by the Shapiro lemma.

The norm compatibility of the $u_{n}$ implies that the homomorphisms

$$
f_{n}(h):=\left(\sqrt[l^{n}]{u_{n}}\right)^{h-1} \in H^{1}\left(H_{n}, \mu_{l^{n}}\right)
$$

are compatible under the corestriction maps, and equation (36) implies that

$$
z(u):=\left(l\left(f_{n}\right)\right)_{n} \in{\underset{\lim }{n}}^{1} Z^{1}\left(\Gamma_{n}, \mathscr{F}_{n}\right) \cong Z^{1}\left(G_{\Sigma}, T_{l}^{\infty}\right)
$$

does in fact lie in $K$. Note that the elements $l\left(f_{n}\right)$ are norm-compatible by Lemma 4.2 applied to $H^{\prime}=\operatorname{Gal}\left(F_{m_{0} l^{n}} / L_{m_{0} l^{n-1}}\right)$ and $M^{\prime}=\mu_{l^{n-1}}$, and that there is an isomorphism of $\Gamma_{n}$-modules $\operatorname{Hom}_{\mathbb{Z}\left[H_{n-1}\right]}\left(\mathbb{Z}\left[\Gamma_{n-1}\right], M^{\prime}\right) \cong \operatorname{Hom}_{\mathbb{Z}\left[H^{\prime}\right]}\left(\mathbb{Z}\left[\Gamma_{n}\right], M^{\prime}\right)$. From now on we denote by $z(u)$ this particular lift of $u$ (depending on a choice of sets of representatives $S_{n}$ formed for increasing $n$ as in Lemma 4.2) and define $\lambda_{\infty}$ by $(c-1) z(u)=d\left(\lambda_{\infty} \sigma\right)$.

Let $\psi_{n} \in \operatorname{Hom}_{\mathbb{Z}\left[H_{n}\right]}\left(\mathbb{Z}\left[\Gamma_{n}\right], \mu_{l^{n}}\right)$ be the element associated to $f_{n}$ by Lemma 4.3. Since $s \phi_{\infty} \in S_{n}$ for any $s \in S_{n}$, we have

$$
\phi_{\infty} s=h_{\phi_{\infty}, s} s \phi_{\infty}
$$


and hence by Lemma 4.3

$$
\psi_{n}(x)=c f_{n}\left(h_{c, x}\right)=c\left(\sqrt[I^{n}]{u_{n}}\right)^{\phi_{\infty} s(x) \phi_{\infty} s(x)^{-1}-1}=\left(\sqrt[l^{n}]{u_{n}}\right)^{s(x)} \phi_{\infty} s(x)^{-1}-1
$$

for $x \in G / H=G_{m_{0} l^{n}}$. Now take $x:=\tau_{a}^{-1}=\tau_{a, m_{0} l^{n}}^{-1}$. Since we are only interested in the value of $\psi_{n}$ "modulo 2 ", i.e. in its $l^{n-1}$-st power, it suffices to compute the action of $s(x) \phi_{\infty} s(x)^{-1}$ on a fixed square root $w_{n} \in F_{m_{0} l^{n}}$ of $u_{n}$. The element $w_{n}^{s(x)^{-1}}$ is a square root of $u_{n}^{\tau_{a}}$ and

$$
w_{n}^{\phi_{\infty} s(x)^{-1}}= \pm w_{n}^{s(x)^{-1}}
$$

according to whether $\sigma_{m_{0} l^{n}}\left(u_{n}^{\tau_{a}}\right)$ is positive or negative. Hence

$$
w_{n}^{s(x) \phi_{\infty} s(x)^{-1}}=(-1)^{\varepsilon_{n, a}} w_{n}
$$

and $\psi_{n}\left(\tau_{a, m_{0} l^{n}}^{-1}\right)^{l^{n-1}}=(-1)^{\varepsilon_{n, a}}$. Writing $\psi_{n}=\lambda_{n}^{\prime} \sigma_{m_{0} l^{n}}$ as in (37), we have

$$
\lambda_{n}^{\prime}=\sum_{g \in G_{m_{0} l^{n}}} \hat{\psi}_{n}(g) g \equiv \sum_{a \bmod m_{0} l^{n}} \varepsilon_{n, a} \tau_{a, m_{0} l^{n}}^{-1} \bmod 2 .
$$

We did not verify that the $\psi_{n}$ are norm-compatible, so we do not know a priori that $\lambda_{n}=\lambda_{n}^{\prime}$ (note that $\lambda_{n} \sigma_{m_{0} l^{n}}$ is the unique norm compatible choice of elements as in Lemma 4.3). However, using the norm-compatibility of $u_{n}$, the explicit definition of $\varepsilon_{n, a}$ and (40) one immediately verifies that the images $\bar{\lambda}_{n}^{\prime}$ of $\lambda_{n}^{\prime}$ in $\mathbb{Z} / 2 \mathbb{Z}\left[G_{m_{0} l^{n}}\right]$ are norm compatible. Hence $\delta_{n}:=\bar{\lambda}_{n}^{\prime}-\bar{\lambda}_{n}$ is norm compatible and, since $d\left(\delta_{n}\right)=0$, for any $n$ we have $\delta_{n} \in\left\{0, N_{n}\right\}$ where $N_{n}=\sum_{g \in G_{m_{0}} l^{n}} g$. Since the norm of $N_{n}$ is zero for $n \geqq 2$ the only norm compatible choice is $\delta_{n}=0$ and we conclude that $\lambda_{n}$ satisfies the required congruence.

The following proposition is the heart of the proof of Theorem 1.2.

Proposition 3.5. There exists a $\Lambda_{\mathfrak{q}}$-basis $z_{m_{0}}$ of $K_{\mathfrak{q}}$ so that

$$
(c-1) z_{m_{0}} \equiv d\left(\left(\gamma-\chi_{\text {cyclo }}(\gamma)\right) \theta_{m_{0}} \sigma\right) \quad \bmod 2(c-1) d\left(T_{l}^{\infty}\right)
$$

and

$$
\partial\left(z_{m_{0}}\right)=v \cdot\left(\gamma-\chi_{\text {cyclo }}(\gamma)\right) \kappa^{\#}\left(\theta_{m_{0}}\right) \cdot \beta_{m_{0}}^{\infty},
$$

where

$$
v=-\frac{1}{m_{0}} \prod_{p \mid m_{0}}\left(\frac{e_{p}\left(1-\mathrm{Fr}_{p}\right)}{e_{p}\left(1-\frac{\mathrm{Fr}_{p}}{p}\right)} v_{p}\right)
$$

with $e_{p}$ defined in (15) and $v_{p}$ defined in Proposition 2.2.

Proof. We define

$$
z_{m_{0}}:=z\left(\eta_{m_{0}}^{\gamma-\chi_{\text {cyclo }}(\gamma)}\right)=z\left(\frac{1-\zeta_{m_{0} l^{n}}^{M}}{\left(1-\zeta_{m_{0} l^{n}}\right)^{M}}\right)_{n},
$$


where $M=\chi_{\text {cyclo }}(\gamma)=1+4 m_{0}$ and $z(u)$ was defined in (39). Note here that $\gamma-\chi_{\text {cyclo }}(\gamma)$ is a unit in $\Lambda_{\mathfrak{q}}$ and that $\eta_{m_{0}}$ is a $\Lambda_{\mathfrak{q}}$-generator of $H^{1}\left(G_{\Sigma}, T_{l}^{\infty}\right)_{\mathfrak{q}}$ so that $z_{m_{0}}$ is indeed a basis of $K_{\mathfrak{q}}$ by Nakayama's lemma.

Then $\partial\left(z_{m_{0}}\right)=\overline{\operatorname{res}}_{l}\left(z_{m_{0}}\right)$ is given by Proposition 2.2. Note that for $m_{0}=1$ the factor $\gamma-1$ in Proposition 2.2 can be canceled since $\gamma-1 \in \Lambda_{\mathfrak{q}}^{\times}$. Defining $\lambda_{\infty}$ by $(c-1) z_{m_{0}}=d\left(\lambda_{\infty} \sigma\right)$, we deduce from Lemmas 3.4 and 3.6 below

$$
\lambda_{n, a} \equiv\left\lfloor\frac{M a}{m_{0} l^{n}}\right\rfloor \bmod 2
$$

On the other hand,

$$
\begin{aligned}
\left(\gamma-\chi_{\text {cyclo }}(\gamma)\right) g_{m_{0} l^{n}} & =-\sum_{a}\left(\frac{a}{m_{0} l^{n}}-\frac{1}{2}\right) \gamma \tau_{a}^{-1}+\sum_{a}\left(\frac{M a}{m_{0} l^{n}}-\frac{M}{2}\right) \tau_{a}^{-1} \\
& =-\sum_{a}\left(\left\langle\frac{M a}{m_{0} l^{n}}\right\rangle-\frac{1}{2}\right) \tau_{a}^{-1}+\sum_{a}\left(\frac{M a}{m_{0} l^{n}}-\frac{M}{2}\right) \tau_{a}^{-1} \\
& =\sum_{a}\left(\left\lfloor\frac{M a}{m_{0} l^{n}}\right\rfloor+\frac{1-M}{2}\right) \tau_{a}^{-1} \\
& \equiv \sum_{a}\left\lfloor\frac{M a}{m_{0} l^{n}}\right\rfloor \tau_{a}^{-1} \bmod 2,
\end{aligned}
$$

where the sums are over $0<a<m_{0} l^{n},\left(a, \operatorname{lm}_{0}\right)=1$ and $\langle x\rangle=x-\lfloor x\rfloor$. Hence

$$
\left(\gamma-\chi_{\text {cyclo }}(\gamma)\right) \theta_{m_{0}}=\left(\left(\gamma-\chi_{\text {cyclo }}(\gamma)\right) g_{m_{0} l^{n}}\right)_{n} \equiv\left(\sum_{a} \lambda_{n, a} \tau_{a}^{-1}\right)_{n}=\lambda_{\infty} \bmod 2
$$

Lemma 3.6. Let $M \equiv 1 \bmod 4$ be an integer, $0<x<1$ a real number and $u=\exp (2 \pi i x)$. Then the sign of the real number $\frac{1-u^{M}}{(1-u)^{M}}$ is $(-1)^{\lfloor M x\rfloor}$.

Proof. For $0<x<1$ the zeros of the real valued differentiable function $f(x)=\frac{1-u^{M}}{(1-u)^{M}}$ are at $x=\frac{1}{M}, \frac{2}{M}, \ldots, \frac{M-1}{M}$ and all of these are simple. Hence $f$ $(1-u)^{M}$ changes sign precisely at those arguments and so does $(-1)^{\lfloor M x\rfloor}$. For $x=\frac{1}{2}$ we have $f(x)=2 / 2^{M}>0$ and $(-1)^{\lfloor M x\rfloor}=(-1)^{\frac{M-1}{2}}=1$, so the signs of the two functions agree.

3.4. Passing from $\Delta^{\infty}$ to $R \Gamma_{c}\left(\mathbb{Z}\left[\frac{1}{m_{0} l}\right], T_{l}^{\infty}\right)$. Recall that the two complexes are
related by

$$
\Delta^{\infty, *}[-3] \otimes_{\Lambda, \kappa^{\#}} \Lambda \cong R \Gamma_{c}\left(\mathbb{Z}\left[\frac{1}{m_{0} l}\right], T_{l}^{\infty}\right),
$$


where $P^{*}=R \operatorname{Hom}_{\Lambda}(P, \Lambda)$ denotes the $\Lambda$-dual and $\kappa^{\#}: \Lambda \rightarrow \Lambda$ is the automorphism $g \mapsto \chi_{\text {cyclo }}(g) g^{-1}$. For $i=1,2$, we denote by $\gamma_{i}^{*}$ the dual basis to $\gamma_{i}$ and by $\gamma_{i}^{* \#}$ the image of $\gamma_{i}^{*}$ in $\Delta^{\infty, i, *} \otimes_{\Lambda, \kappa^{*}} \Lambda$. By Proposition 3.2 we may pick isomorphisms

$$
\left(\Delta^{\infty, 2, *} \otimes_{\Lambda, \kappa^{\#}} \Lambda\right)_{\mathfrak{q}} \cong K_{\mathfrak{q}}, \quad\left(\Delta^{\infty, 1, *} \otimes_{\Lambda, \kappa^{*}} \Lambda\right)_{\mathfrak{q}} \cong H^{1}\left(\mathbb{Q}_{l}, T_{l}^{\infty}\right)_{\mathfrak{q}}
$$

and the following proposition summarizes the information we have about $\gamma_{2}^{* \#}$ from the defining properties of $\gamma_{1}, \gamma_{2}$ and $\alpha$.

Proposition 3.7. We have

$$
(c-1) \gamma_{2}^{* \#}=(c-1) \sigma
$$

and

$$
\partial\left(\gamma_{2}^{* \#}\right)=\kappa^{\#}(\alpha) \kappa^{\#}(v \varrho)^{-1} \cdot\left(\frac{\theta_{m_{0}}}{1-c}\right)^{-1} \cdot \beta_{m_{0}}^{\infty}
$$

where $v($ resp. $\varrho)$ was defined in (41) (resp. (12)) and $\frac{\theta_{m_{0}}}{1-c}$ denotes any $x \in \Lambda_{\mathfrak{q}}^{\times}$satisfying $(1-c) x=\theta_{m_{0}}$.

Proof. We may pick a $\Lambda_{\mathfrak{q}}$-isomorphism $\Delta_{\mathfrak{q}}^{\infty, 1} \cong H^{1}\left(\mathbb{Q}_{l}, T_{l}^{\infty}\right)_{\mathfrak{q}}$ so that the diagram

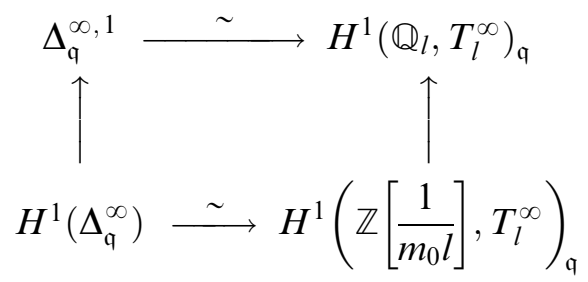

commutes. If we set

$$
\gamma_{1}:=v \cdot \frac{\kappa^{\#}\left(\theta_{m_{0}}\right)}{1+c} \cdot \beta_{m_{0}}^{\infty}
$$

where $v$ was defined in (41) then $\gamma_{1}$ satisfies the defining property $(1+c) \gamma_{1}=\eta_{m_{0}}$ by Proposition 2.2. Hence

$$
\gamma_{1}^{*}=v^{-1} \cdot\left(\frac{\kappa^{\#}\left(\theta_{m_{0}}\right)}{1+c}\right)^{-1} \cdot \beta_{m_{0}}^{\infty, *}
$$

and

$$
\gamma_{1}^{* \#}=\kappa^{\#}(v)^{-1} \cdot\left(\frac{\theta_{m_{0}}}{1-c}\right)^{-1} \cdot \beta_{m_{0}}^{\infty, * \#}=\kappa^{\#}(v)^{-1} \cdot\left(\frac{\theta_{m_{0}}}{1-c}\right)^{-1} \cdot \kappa^{\#}(\varrho)^{-1} \cdot \beta_{m_{0}}^{\infty}
$$


since $\left\langle\beta_{m_{0}}^{\infty}, \kappa^{\#}(\varrho)^{-1} \beta_{m_{0}}^{\infty}\right\rangle=1$ by the definition of $\varrho$ in (12). Now $\partial^{\prime}\left(\gamma_{1}\right)=\alpha \gamma_{2}$ implies $\partial\left(\gamma_{2}^{*}\right)=\alpha \gamma_{1}^{*}$ and

$$
\partial\left(\gamma_{2}^{* \#}\right)=\kappa^{\#}(\alpha) \gamma_{1}^{* \#}=\kappa^{\#}(\alpha) \kappa^{\#}(v \varrho)^{-1} \cdot\left(\frac{\theta_{m_{0}}}{1-c}\right)^{-1} \cdot \beta_{m_{0}}^{\infty}
$$

noting that $\partial^{\prime}$ and $\partial$ are dual maps. This finishes the proof of (43). Concerning (42) we have a commutative diagram

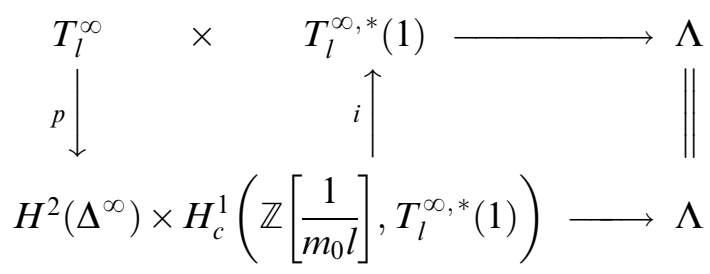

where $i$ is the inclusion

$$
H_{c}^{1}\left(\mathbb{Z}\left[\frac{1}{m_{0} l}\right], T_{l}^{\infty, *}(1)\right) \cong H^{0}\left(\mathbb{R}, T_{l}^{\infty, *}(1)\right)=T_{l}^{\infty, *}(1)^{\phi_{\infty}=1} \rightarrow T_{l}^{\infty, *}(1) .
$$

The defining property of $\gamma_{2}$ that $p\left(\gamma_{2}\right)=\sigma$ translates into $(c+1) \gamma_{2}^{*}=(c+1) \sigma$ and $(c-1) \gamma_{2}^{* \#}=(c-1) \sigma$.

Denote by $J \subseteq \mathfrak{q} \Lambda_{\mathfrak{q}}$, the $\Lambda_{\mathfrak{q}}$-ideal

$$
J:=(c+1, c-1)=(2, c-1)=(2, c+1) .
$$

By Proposition 3.5 the basis

$$
z:=\left(\gamma-\chi_{\text {cyclo }}(\gamma)\right)^{-1}\left(\frac{\theta_{m_{0}}}{c-1}\right)^{-1} z_{m_{0}}
$$

of $K_{\mathfrak{q}}$ satisfies

$$
(c-1) z \equiv(c-1) \sigma=(c-1) \gamma_{2}^{* \#} \bmod J(c-1) T_{l}^{\infty},
$$

hence

$$
z \equiv \gamma_{2}^{* \#} \bmod J K_{\mathrm{q}} .
$$

Since $z$ is a basis of $K_{\mathfrak{q}}$ we can write $z(1+j)=\gamma_{2}^{* \#}$ with $j \in J$. By Proposition 3.5 we have

$$
\partial(z)=v \cdot\left(\frac{\theta_{m_{0}}}{c-1}\right)^{-1} \cdot \kappa^{\#}\left(\theta_{m_{0}}\right) \cdot \beta_{m_{0}}^{\infty}
$$

and comparing this with $\partial\left(\gamma_{2}^{* \#}\right)$ in Proposition 3.7 we find

$$
\kappa^{\#}(\alpha)=-\kappa^{\#}(v \varrho) \cdot v \cdot \kappa^{\#}\left(\theta_{m_{0}}\right) \cdot(1+j)
$$


or, equivalently,

$$
\begin{aligned}
\alpha & =-v \cdot \varrho \cdot \kappa^{\#}(v) \cdot \theta_{m_{0}} \cdot\left(1+\kappa^{\#}(j)\right) \\
& =\theta_{m_{0}} \cdot \kappa^{\#}(v) \cdot v \cdot \varrho \cdot\left(1+j_{1}\right)
\end{aligned}
$$

with $j_{1}=-2-\kappa^{\#}(j) \in J$. Recall from Lemma 3.1 that we need to show that $\alpha=\theta_{m_{0}}\left(1+j_{2}\right)$ for some $j_{2} \in J$. This is then accomplished by the following lemma:

Lemma 3.8. For $v$ defined in (41), @ defined in (12) and $J$ defined in (44) we have

$$
\kappa^{\#}(v) \cdot v \cdot \varrho \in 1+J
$$

Proof. First note that

$$
\kappa^{\#}\left(e_{I_{p}}\right)=\frac{1}{\left|I_{p}\right|} \sum_{g \in I_{p}} \kappa^{\#}(g)=\frac{1}{\left|I_{p}\right|} \sum_{g \in I_{p}} g^{-1}=e_{I_{p}}
$$

and therefore

$$
\kappa^{\#} e_{p}(x)=\kappa^{\#}\left(1+(x-1) e_{I_{p}}\right)=1+\left(\kappa^{\#}(x)-1\right) e_{I_{p}}=e_{p}\left(\kappa^{\#}(x)\right) .
$$

Moreover

$$
\kappa^{\#}\left(\operatorname{Fr}_{p}\right)=\chi_{\text {cyclo }}\left(\operatorname{Fr}_{p}\right) \operatorname{Fr}_{p}^{-1}=p / \operatorname{Fr}_{p}
$$

and

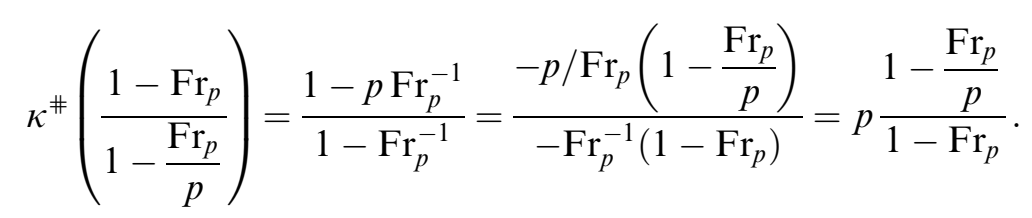

Hence

$$
\kappa^{\#}(v) \cdot v \cdot \varrho=\frac{1}{m_{0}^{2}} \varrho \prod_{p \mid m_{0}} e_{p}(p) \kappa^{\#}\left(v_{p}\right) v_{p} .
$$

Since $m_{0}$ is an odd integer we have $m_{0}^{-2} \in 1+J$ and it suffices to show that $\kappa^{\#}\left(v_{p}\right) v_{p} \in 1+J$ and that $\varrho \prod e_{p}(p) \in 1+J$. Now $\kappa^{\#}\left(v_{p}\right) v_{p} \in \Lambda^{\times}$is associated via Lemma 2.1 to the function $\quad p \mid m_{0}$

$$
u: x \mapsto\left(p / \widetilde{\operatorname{Fr}}_{p} \cdot \widetilde{\operatorname{Fr}}_{p}\right)^{\max (x, 1)}=p^{\max (x, 1)}
$$

that is

$$
\kappa^{\#}\left(v_{p}\right) v_{p}=\sum_{\chi} u\left(\operatorname{ord}_{p}\left(f_{\chi}\right)\right) e_{\chi}=1+2 \sum_{\chi} v\left(\operatorname{ord}_{p}\left(f_{\chi}\right)\right) e_{\chi} \in 1+J
$$


where $v$ is the function $x \mapsto\left(p^{\max (x, 1)}-1\right) / 2$. Note here that

$$
v(0)-v(1)=(p-1) / 2-(p-1) / 2=0
$$

is indeed divisible by $(p-1)$ in $\Lambda$, so Lemma 2.1 applies.

As to

$$
\varrho \prod_{p \mid m_{0}} e_{p}(p)=c_{l} \varrho_{0} \prod_{p \mid m_{0}} e_{p}(p)
$$

we note that in the sum (13) the term $\phi\left(m_{0}\right) / \phi(d /(d, g+1))$ is odd precisely when all primes dividing $m_{0}$ also divide $d /(d, g+1)$, i.e. precisely when $d=\prod_{p \mid m_{0}} p^{\operatorname{ord}_{p}(g+1)+1}$ (and $\left.d \mid m_{0}\right)$. So modulo 2 the sum (13) reduces to one nonzero term if $g+\stackrel{p \mid m_{0}}{1}=0$ mod $p^{\operatorname{ord}_{p}\left(m_{0}\right)}$ for all $p \mid m_{0}$, and has all terms equal to zero if there is a $p \mid m_{0}$ with $g+1 \equiv 0 \bmod p^{\operatorname{ord}_{p}\left(m_{0}\right)}$. This implies

$$
\varrho_{0}=\sum_{g \in G_{m_{0}}} \varrho_{0}(g) \cdot g^{-1} \equiv \prod_{p \mid m_{0}}\left(\sum_{\substack{g \in I_{p} \\ g \neq c_{p}}} g\right) \equiv \prod_{p \mid m_{0}}\left(c_{p}+\tilde{e}_{I_{p}}\right) \quad \bmod 2
$$

where $c_{p}$ is the projection of $c$ to $I_{p}$ and $\tilde{e}_{I_{p}}=\left|I_{p}\right| e_{I_{p}}=\sum_{g \in I_{p}} g$. Since

$$
e_{p}(p)=1+(p-1) e_{I_{p}} \equiv 1+\tilde{e}_{I_{p}} \bmod 2
$$

we deduce

$$
\begin{aligned}
\varrho \prod_{p \mid m_{0}} e_{p}(p) & \equiv c_{l} \prod_{p \mid m_{0}}\left(c_{p}+\tilde{e}_{I_{p}}\right)\left(1+\tilde{e}_{I_{p}}\right)=c_{l} \prod_{p \mid m_{0}}\left(c_{p}+2 \tilde{e}_{I_{p}}+\left|I_{p}\right| \tilde{e}_{I_{p}}\right) \\
& \equiv c_{l} \prod_{p \mid m_{0}} c_{p}=c=1+(c-1) \equiv 1 \bmod J
\end{aligned}
$$

This finishes the proof of the lemma.

\section{Appendix. Generalities on the Shapiro lemma}

In this section, we fix a group $G$ with subgroup $H$ and a set $S \subseteq G$ of right coset representatives so that $G=\bigcup_{s \in S} H s$. Modules are understood to be left modules. We assume that $S$ contains the identity and define $h(g) \in H$ and $s(g) \in S$ for $g \in G$ by

$$
g=h(g) s(g)
$$

Then for $h \in H$ and $g \in G$

$$
\begin{aligned}
& \qquad h(h g)=h h(g), \quad s(h g)=s(g) \\
& \text { Brought to you by | California Institute of Technology (California Institute of Technology) } \\
& \text { Authenticated | 172.16.1.226 } \\
& \text { Download Date | 1/20/12 4:57 PM }
\end{aligned}
$$


If $H$ is normal in $G$ we define for $g_{1}, g_{2} \in G$

$$
h_{g_{1}, g_{2}}:=\left({ }^{s\left(g_{1}\right)} h\left(g_{2}\right)\right)^{-1} h\left(g_{1}\right)^{-1} h\left(g_{1} g_{2}\right) \in H
$$

where ${ }^{a} b=a b a^{-1}$. Then one verifies that

$$
s\left(g_{1}\right) s\left(g_{2}\right)=h_{g_{1}, g_{2}} s\left(g_{1} g_{2}\right)
$$

which together with (45) implies that $h_{g_{1}, g_{2}}$ only depends on the classes of $g_{1}, g_{2}$ in $G / H$. The function $h_{g_{1}, g_{2}}$ satisfies

$$
h_{g_{1}, g_{2}} h_{g_{1} g_{2}, g_{3}}={ }^{s\left(g_{1}\right)} h_{g_{2}, g_{3}} h_{g_{1}, g_{2} g_{3}}
$$

which is the standard 2-cocycle relation if $H$ is abelian.

For any $H$-module $M$ we let

$$
\operatorname{Coind}_{H}^{G} M:=\operatorname{Hom}_{\mathbb{Z}[H]}(\mathbb{Z}[G], M)
$$

be the coinduced module with its natural left $G$-action given by

$$
(g \psi)(x)=\psi(x g) .
$$

We denote by $\mathbb{Z}\left[G^{\bullet}\right] \rightarrow \mathbb{Z}$ the standard resolution, and similarly for $H$.

Consider the Shapiro-lemma quasi-isomorphism

$$
\begin{gathered}
\operatorname{Hom}_{\mathbb{Z}[G]}\left(\mathbb{Z}\left[G^{\bullet}\right], \operatorname{Hom}_{\mathbb{Z}[H]}(\mathbb{Z}[G], M)\right) \cong \operatorname{Hom}_{\mathbb{Z}[H]}\left(\mathbb{Z}\left[G^{\bullet}\right], M\right) \\
\stackrel{\rho}{\rightarrow} \operatorname{Hom}_{\mathbb{Z}[H]}\left(\mathbb{Z}\left[H^{\bullet}\right], M\right)
\end{gathered}
$$

where the first isomorphism is the canonical adjunction and $\rho$ is given by restriction of cochains to $H$. The map $\rho$ has a section $l$ induced by the homomorphism $\mathbb{Z}\left[G^{n}\right] \rightarrow \mathbb{Z}\left[H^{n}\right]$, $\left(g_{1}, \ldots, g_{n}\right) \mapsto\left(h\left(g_{1}\right), \ldots, h\left(g_{n}\right)\right)$ which is $H$-equivariant by $(45)$ and which depends on the choice of $S$.

Lemma 4.1. The map $\mathrm{l}$ induces a map

$$
\imath: C^{1}(H, M) \rightarrow C^{1}\left(G, \operatorname{Coind}_{H}^{G} M\right)=C^{1}\left(G, \operatorname{Hom}_{\mathbb{Z}[H]}(\mathbb{Z}[G], M)\right)
$$

on inhomogeneous 1-cochains which for $g, x \in G$ is given by

$$
(l(f)(g))(x)=h(x) f\left(h(x)^{-1} h(x g)\right)=h(x) f\left({ }^{s(x)} h(g) h_{x, g}\right) .
$$

This last expression holds if $H$ is normal in $G$.

Proof. To the inhomogeneous 1-cochain $f$ with values in $M$ there is associated, respectively, a homogeneous cochain on $H$, its image under $l$ on $G$, its image under the 
adjunction with values in $\operatorname{Hom}_{\mathbb{Z}[H]}(\mathbb{Z}[G], M)$ and its image by passing to inhomogeneous cochains on $G$ as follows:

$$
\begin{aligned}
\left(h_{1}, h_{2}\right) & \mapsto h_{1} f\left(h_{1}^{-1} h_{2}\right), \\
\left(g_{1}, g_{2}\right) & \mapsto h\left(g_{1}\right) f\left(h\left(g_{1}\right)^{-1} h\left(g_{2}\right)\right), \\
\left(g_{1}, g_{2}\right) & \mapsto\left(x \mapsto h\left(x g_{1}\right) f\left(h\left(x g_{1}\right)^{-1} h\left(x g_{2}\right)\right)\right), \\
g & \mapsto\left(x \mapsto h(x) f\left(h(x)^{-1} h(x g)\right)\right) .
\end{aligned}
$$

The second identity follows from (46).

Now let $H \subseteq H^{\prime} \subseteq G$ be a larger subgroup so that $\left[H^{\prime}: H\right]<\infty, S^{\prime}$ (resp. $E$ ) a set of representatives of $H^{\prime} \backslash G$ (resp. $H \backslash H^{\prime}$ ) and $S=E S^{\prime}$. Denote by $h^{\prime}, s^{\prime}$ and $l^{\prime}$ the maps $h, s$ and $\imath$ formed with respect to the set $S^{\prime}$.

Lemma 4.2. Let $\operatorname{tr}_{H^{\prime} / H}=\sum_{t \in E} t^{-1}$ be the trace operator on any left $H^{\prime}$-module, let $M^{\prime}$ be a $H^{\prime}$-module with trivial action and let

$$
H^{1}\left(H, M^{\prime}\right)=\operatorname{Hom}\left(H, M^{\prime}\right)=Z^{1}\left(H, M^{\prime}\right) \subseteq C^{1}\left(H, M^{\prime}\right)
$$

be the group of one-cocycles. Then there is a commutative diagram

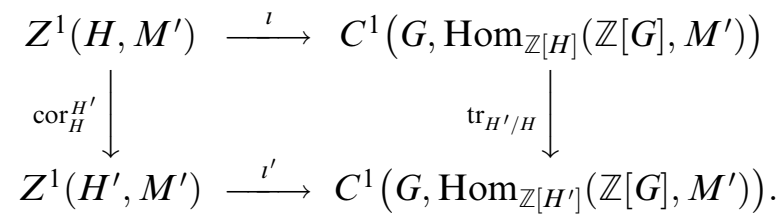

Proof. The first isomorphism in (49) fits in a commutative diagram

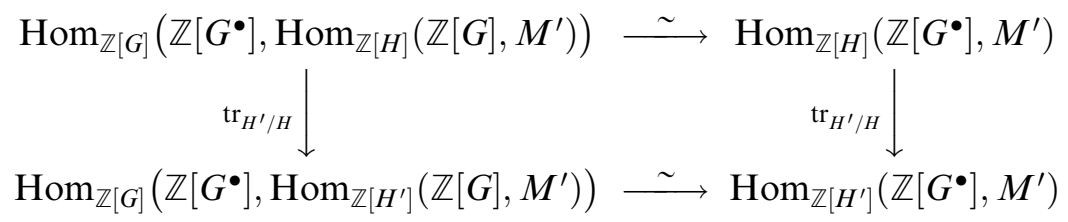

which arises from the trace map $\operatorname{tr}_{H^{\prime} / H}: N^{H} \rightarrow N^{H^{\prime}}$ applied to the $H^{\prime}$-module

$$
\operatorname{Hom}_{\mathbb{Z}[G]}\left(\mathbb{Z}\left[G^{\bullet}\right], \operatorname{Hom}_{\mathbb{Z}}\left(\mathbb{Z}[G], M^{\prime}\right)\right) \stackrel{\sim}{\rightarrow} \operatorname{Hom}_{\mathbb{Z}}\left(\mathbb{Z}\left[G^{\bullet}\right], M^{\prime}\right)
$$

There is a commutative diagram

$$
\begin{aligned}
& \operatorname{Hom}_{\mathbb{Z}[H]}\left(\mathbb{Z}\left[G^{\bullet}\right], M^{\prime}\right) \stackrel{l_{2}}{\longleftarrow} \operatorname{Hom}_{\mathbb{Z}[H]}\left(\mathbb{Z}\left[H^{\prime \bullet}\right], M^{\prime}\right) \stackrel{l_{1}}{\longleftarrow} \operatorname{Hom}_{\mathbb{Z}[H]}\left(\mathbb{Z}\left[H^{\bullet}\right], M^{\prime}\right) \\
& \operatorname{tr}_{H^{\prime} / H} \downarrow \quad \operatorname{tr}_{H^{\prime} / H} \downarrow \\
& \operatorname{Hom}_{\mathbb{Z}\left[H^{\prime}\right]}\left(\mathbb{Z}\left[G^{\bullet}\right], M^{\prime}\right) \stackrel{l^{\prime}}{\longleftarrow} \operatorname{Hom}_{\mathbb{Z}\left[H^{\prime}\right]}\left(\mathbb{Z}\left[H^{\prime \bullet}\right], M^{\prime}\right) \\
& \text { Brought to you by | California Institute of Technology (California Institute of Technology) }
\end{aligned}
$$


where the maps $l^{\prime}$ and $l_{2}$ are induced by $\mathbb{Z}\left[G^{n}\right] \rightarrow \mathbb{Z}\left[H^{\prime n}\right]$ sending $\left(g_{1}, \ldots, g_{n}\right)$ to $\left(h^{\prime}\left(g_{1}\right), \ldots, h^{\prime}\left(g_{n}\right)\right)$. The commutativity follows from (45): For $t \in E \subseteq H^{\prime}$ we have $t^{-1} \phi\left(t h^{\prime}\left(g_{1}\right), \ldots, t h^{\prime}\left(g_{n}\right)\right)=t^{-1} \phi\left(h^{\prime}\left(t g_{1}\right), \ldots, h^{\prime}\left(t g_{n}\right)\right)$. Finally, the corestriction map on $H^{1}(H, M)$ is induced by the trace map $\operatorname{tr}_{H^{\prime} / H}$ on $\operatorname{Hom}_{\mathbb{Z}[H]}\left(\mathbb{Z}\left[H^{\prime \bullet}\right], M^{\prime}\right)$ ([2], Chapter III.9 (C)). The quasi-isomorphism $l_{1}$ is induced by $\mathbb{Z}\left[H^{\prime n}\right] \rightarrow \mathbb{Z}\left[H^{n}\right]$ sending $\left(g_{1}, \ldots, g_{n}\right)$ to $\left(h\left(g_{1}\right), \ldots, h\left(g_{n}\right)\right)$ and we have $l=l_{2} \circ l_{1}$ since $h\left(h^{\prime}(g)\right)=h(g)$ (this follows from $\left.S=E S^{\prime}\right)$.

We now assume that $H$ is normal in $G$ with quotient $Q=G / H$ and that $M$ is a $G$-module. Then there is a natural left $Q$-action on the coinduced module

$$
\operatorname{Coind}_{H}^{G} \operatorname{Res}_{H}^{G} M=\operatorname{Hom}_{\mathbb{Z}[H]}(\mathbb{Z}[G], M)
$$

given by

$$
(q \psi)(x)=\tilde{q} \psi\left(\tilde{q}^{-1} x\right)
$$

where $\tilde{q} \in G$ is any lift. This action commutes with the left $G$-action (48) and leads to the natural $\mathbb{Z}[Q]$-module structure on $H^{i}\left(G, \operatorname{Coind}_{H}^{G} M\right) \cong H^{i}(H, M)$. The first isomorphism in $(49)$ is $\mathbb{Z}[Q]$-equivariant where $q \in Q$ acts on $\operatorname{Hom}_{\mathbb{Z}[H]}\left(\mathbb{Z}\left[G^{\bullet}\right], M\right)$ via

$$
(q f)\left(x_{1}, \ldots, x_{n}\right):=\tilde{q} f\left(\tilde{q}^{-1} x_{1}, \ldots, \tilde{q}^{-1} x_{n}\right) .
$$

The restriction map $\rho$ and its section $l$ are only $\mathbb{Z}[Q]$-equivariant up to homotopy where $\operatorname{Hom}_{\mathbb{Z}[H]}\left(\mathbb{Z}\left[H^{\bullet}\right], M\right)$ is given its natural $Q$-action

$$
(q f)\left(x_{1}, \ldots, x_{n}\right):=\tilde{q} f\left(\tilde{q}^{-1} x_{1} \tilde{q}, \ldots, \tilde{q}^{-1} x_{n} \tilde{q}\right) .
$$

Lemma 4.3. Assume that $H \unlhd G$ and that $M$ is a $G$-module with trivial H-action. Let

$$
f \in H^{1}(H, M)=\operatorname{Hom}(H, M)=Z^{1}(H, M)
$$

be a cohomology class fixed by $c \in Q=G / H$. Then

$$
(c-1) l(f)=d(\psi)
$$

where $\psi \in \operatorname{Coind}_{H}^{G} M:=\operatorname{Hom}_{\mathbb{Z}[H]}(\mathbb{Z}[G], M)$ is given for $x \in G$ by

$$
\psi(x):=c f\left(h_{c^{-1}, x}\right)
$$

and

$$
d: \operatorname{Coind}_{H}^{G} M \rightarrow C^{1}\left(G, \operatorname{Coind}_{H}^{G} M\right)
$$

is the differential in the inhomogeneous standard complex.

Proof. The fact that $f$ is fixed by $c$ translates into the identity $f\left({ }^{s(c)} h\right)=c f(h)$ (and the same for $c$ replaced by $c^{-1}$ and we could have chosen any representative for $c$ in $G$ in place of $s(c)$ ). From Lemma 4.1 and the fact that $H$ acts trivially on $M$ one has 


$$
\begin{aligned}
((c-1) l(f)(g))(x) & =c f\left({ }^{s\left(c^{-1} x\right)} h(g) h_{c^{-1} x, g}\right)-f\left({ }^{s(x)} h(g) h_{x, g}\right) \\
& =c f\left({ }^{s\left(c^{-1} x\right)} h(g)\right)-f\left({ }^{s(x)} h(g)\right)+c f\left(h_{c^{-1} x, g}\right)-f\left(h_{x, g}\right) \\
& =c f\left({ }^{k s\left(c^{-1}\right) s(x)} h(g)\right)-f\left({ }^{s(x)} h(g)\right)+c f\left(h_{c^{-1} x, g}\right)-f\left(h_{x, g}\right) \\
& =c f\left(h_{c^{-1} x, g}\right)-f\left(h_{x, g}\right)
\end{aligned}
$$

where $k=h_{c^{-1}, x}^{-1} \in H$ so that $f\left({ }^{k} h\right)=h$ for all $h \in H$. On the other hand, applying $f$ to (47) with $g_{1}=c^{-1}, \stackrel{c}{g}_{2}=x, g_{3}=g$ we find

$$
\begin{aligned}
f\left(h_{c^{-1}, x}\right)+f\left(h_{c^{-1} x, g}\right) & =f\left({ }^{s\left(c^{-1}\right)} h_{x, g}\right)+f\left(h_{c^{-1}, x g}\right) \\
& =c^{-1} f\left(h_{x, g}\right)+f\left(h_{c^{-1}, x g}\right)
\end{aligned}
$$

or equivalently

$$
f\left(h_{c^{-1} x, g}\right)-c^{-1} f\left(h_{x, g}\right)=f\left(h_{c^{-1}, x g}\right)-f\left(h_{c^{-1}, x}\right) .
$$

Multiplying with $c$ and combining with (51) we get

$$
((c-1) l(f)(g))(x)=c f\left(h_{c^{-1}, x g}\right)-c f\left(h_{c^{-1}, x}\right)=(d \psi)(x)
$$

\section{References}

[1] S. Bloch and K. Kato, L-functions and Tamagawa numbers of motives, in: The Grothendieck Festschrift I, Progr. Math. 86 (1990), 333-400.

[2] K. S. Brown, Cohomology of groups, Grad. Texts Math. 87, Springer, New York 1982.

[3] D. Burns, Congruences between derivatives of abelian $L$-functions at $s=0$, Invent. Math. 169 (2007), 451-499.

[4] D. Burns and M. Flach, On Galois structure invariants associated to Tate motives, Amer. J. Math. 120 (1998), 1343-1397.

[5] D. Burns and M. Flach, Tamagawa numbers for motives with (non-commutative) coefficients II, Amer. J. Math. 125 (2003), no. 3, 475-512.

[6] D. Burns and M. Flach, On the equivariant Tamagawa number conjecture for Tate motives, Part II, Documenta Math., Extra Volume: John H. Coates Sixtieth Birthday (2006), 133-163.

[7] D. Burns and C. Greither, On the equivariant Tamagawa number conjecture for Tate motives, Invent. Math. 153 (2003), 303-359.

[8] R. Coleman, The dilogarithm and the norm residue symbol, Bull. Soc. Math. France 109 (1981), $373-402$.

[9] M. Flach, The equivariant Tamagawa number conjecture: A survey, in: Stark's conjecture: Recent work and new directions, D. Burns et al., eds., Contemp. Math. 358 (2004).

[10] T. Fukaya and K. Kato, A formulation of conjectures on $p$-adic zeta functions in non-commutative Iwasawa theory, Proc. St. Petersburg Math. Soc. 11 (2005).

[11] R. Gillard, Unités cyclotomiques, unités semi-locales et $\mathbb{Z}_{p}$-extensions, Ann. Inst. Fourier 29, 4 (1979), $1-15$.

[12] C. Greither, Determining Fitting ideals of minus class groups via the equivariant Tamagawa number conjecture, Compos. Math. 143 (2007), 1399-1426.

[13] K. Iwasawa, On some modules in the theory of cyclotomic fields, J. Math. Soc. Japan 16 (1964), 42-82.

[14] K. Kato, Lectures on the approach to Iwasawa theory of Hasse-Weil $L$-functions via $B_{\mathrm{dR}}$, Part I, in: Arithmetical Algebraic Geometry, E. Ballico, ed., Lect. Notes Math. 1553, Springer (1993).

[15] B. Mazur and A. Wiles, Class fields of abelian extensions of $\mathbb{Q}$, Invent. Math. 76 (1984), 179-330.

[16] J. S. Milne, Arithmetic duality theorems, Perspect. Math. 1 (1986). 
[17] B. Perrin-Riou, Théorie d'Iwasawa des représentations p-adiques sur un corps local, Invent. Math. 115 (1994), 81-149.

[18] K. Rubin, The main conjecture, Appendix to: S. Lang, Cyclotomic fields I and II, 1990.

[19] L. C. Washington, Introduction to Cyclotomic fields, 2nd ed., Grad. Texts Math. 83, Springer, New York 1997.

Department of Mathematics, Caltech 253-37, Pasadena, CA 91125, USA

e-mail: flach@caltech.edu

Eingegangen 30. Januar 2009, in revidierter Fassung 20. August 2010 